This item was submitted to Loughborough's Research Repository by the author.

Items in Figshare are protected by copyright, with all rights reserved, unless otherwise indicated.

\title{
Generalized Henon maps: the cubic diffeomorphisms of the plane
}

PLEASE CITE THE PUBLISHED VERSION

\section{LICENCE}

CC BY-NC-ND 4.0

\section{REPOSITORY RECORD}

Dullin, Holger R., and J.D. Meiss. 2019. "Generalized Henon Maps: The Cubic Diffeomorphisms of the Plane". figshare. https://hdl.handle.net/2134/845. 


\title{
Generalized Hénon Maps: the Cubic Diffeomorphisms of the Plane *
}

\author{
H. R. Dullin \\ Department of Mathematical Sciences \\ Loughborough University \\ Loughborough LE11 3TU, UK \\ H.R.Dullin@lboro.ac.uk \\ J. D. Meiss \\ Department of Applied Mathematics \\ University of Colorado \\ Boulder, CO 80309-0526 \\ James.Meiss@Colorado.EDU
}

November 24, 1999

This paper is dedicated to the memory of John David Crawford, from whom we learned much about the beauty of mathematical physics.

\begin{abstract}
In general a polynomial automorphism of the plane can be written as a composition of generalized Hénon maps. These maps exhibit some of the familiar properties of the quadratic Hénon map, including a bounded set of bounded orbits and an anti-integrable limit. We investigate in particular the cubic, area-preserving case, which reduces to two, two-parameter families of maps. The bifurcations of low period orbits of these maps are discussed in detail.
\end{abstract}

Keywords: polynomial diffeomorphisms, Jacobian conjecture, bifurcations, antiintegrable limit

\section{Introduction}

The simplest dynamical systems are maps $x^{\prime}=f(x)$, and the simplest maps are polynomials. Though in one dimension any invertible map has trivial dynamics, this is no

${ }^{*}$ HRD was supported in part by DFG grant Du 302/2. JDM was supported in part by NSF grant DMS-9971760. 
longer true in higher dimensions. The simplest, nontrivial diffeomorphism of the plane is the Hénon map, [1]

$$
\left(x^{\prime}, y^{\prime}\right)=\left(y+x^{2}+a,-b x\right) .
$$

This map has constant Jacobian, $b$, and when $b \neq 0$ is a diffeomorphism. In fact, any quadratic map of the plane with Jacobian $b \neq 0$, can either be put in the form (1) by conjugation with an affine map, or else it has invariant lines and is dynamically trivial. The Hénon map has been extensively studied because of its genericity and its simplicity, which belie the complexity of its dynamics.

Polynomial diffeomorphisms are also of interest as approximations of more complicated maps with constant Jacobian, just as Taylor series provide approximations for functions. For example, a storage ring for a particle accelerator can be modeled as a composition of maps, each corresponding either to a section of the ring or to a focusing or bending magnet. Since the dynamics is (to a good approximation) Hamiltonian, the resulting maps are symplectic. Polynomial maps provide accurate models of storage ring elements in the "thin lens" approximation [2]. A modern storage ring consists of $10^{3}$ to $10^{4}$ elements, so the one-turn map is difficult to evaluate with speed and accuracy. Thus it is an important problem to obtain a reduced description of this system that can be rapidly evaluated but is faithful to the dynamics. One technique, called "Cremona symplectification," is to try to represent the full system by a composition of a small number of symplectic polynomial diffeomorphisms [3].

One step along the way to this reduction is to obtain a normal form for an arbitrary polynomial diffeomorphism of degree $d$. This problem is unsolved; the obstruction being that it is not known in general that a polynomial diffeomorphism of $\mathbb{C}^{n}$ has a polynomial inverse. This is the content of the unproven, but much studied, "Jacobian conjecture" [4]. For the planar case, the structure of the group of polynomial maps with polynomial inverses, the "affine Cremona group," was obtained by Jung in 1942 [5]. More recently Friedland and Milnor [6] showed that any Cremona map of the plane is either conjugate to a composition of generalized Hénon maps,

$$
h(x, y)=(y+p(x),-b x),
$$

where $p(x)$ is a polynomial and $b \neq 0$, or is dynamically trivial. Note that the inverse of of a generalized Hénon map is

$$
h^{-1}(x, y)=(-y / b, x-p(-y / b)) .
$$

If follows that any composition of Hénon maps has an inverse which is polynomial and of the same degree as the composition. We will review these results in $\S 2$.

In this paper we concentrate on the dynamics of a single, generalized Hénon map, leaving the composite case to future work. Some of the general properties of these maps are discussed in $\S 3$. We specialize to the cubic family in $\S 4$. This family is determined by the polynomial $p(x)= \pm x^{3}+l x+k$, so that it comes in two varieties $C_{ \pm}$depending upon the sign of the cubic term. We study the fixed points and low period orbits for these maps, obtain curves in the parameter plane for the codimension one bifurcations, and classify the codimension two bifurcations. 


\section{Polynomial Automorphisms of the Plane}

A polynomial map of the plane has the form

$$
f(x, y)=\left(x^{\prime}, y^{\prime}\right)=(X(x, y), Y(x, y)),
$$

where $X$ and $Y$ are polynomials in $x$ and $y$. We say that the degree of $f$, $\operatorname{deg}(f)=d$, is the maximum of the degrees of $X$ and $Y$. The map has constant Jacobian if

$$
\operatorname{det} J(X, Y)=\frac{\partial X}{\partial x} \frac{\partial Y}{\partial y}-\frac{\partial X}{\partial y} \frac{\partial Y}{\partial x}
$$

is a constant, and is area-preserving if the Jacobian is one. We will call polynomial maps whose Jacobian is nonzero and constant Keller maps, and denote them by $\mathcal{K}$. The structure of the set $\mathcal{K}$ is not entirely understood. Affine maps,

$$
\mathcal{A}=\{a \in \mathcal{K}: a(x, y)=(\xi, \eta)+L(x, y)\}
$$

where $L$ is a nonsingular linear map, form a simple subset of $\mathcal{K}$. Since nonsingularity of $L$ guarantees the invertibility of $a$, these obviously form a group under composition. Another group in $\mathcal{K}$ is $\mathcal{E}$, the set of elementary or triangular maps,

$$
\mathcal{E}=\{e \in \mathcal{K}: e(x, y)=(\alpha x+\xi, \beta y+p(x))\}
$$

for any polynomial $p$, and $\alpha \beta \neq 0$. As dynamical systems, the affine and elementary cases are trivial.

Of course there are dynamically interesting maps in $\mathcal{K}$, including the generalized Hénon maps (2). Recall that any generalized Hénon map has a polynomial inverse, (3). A famous conjecture asserts that this is true for any map in $\mathcal{K}$, i.e.,

Keller's Jacobian Conjecture [7] Every map in $\mathcal{K}$ has a polynomial inverse, and is therefore a diffeomorphism.

A Keller map with a polynomial inverse is called a polynomial automorphism; these form a group, the affine Cremona group which we denote by $\mathcal{C} .{ }^{1}$ Much work has been devoted to the study of Keller's conjecture and its higher dimensional generalization [4]. It was shown by Wang, for example, that a quadratic Keller map in dimension $n$ has a polynomial inverse [9]. It is also known that the degree of the inverse of a Cremona map $f$ is at most $(\operatorname{deg}(f))^{n-1}[7]$. For the plane it is known that every Keller map with prime degree less than 100 is a Cremona map [10]. It turns out that to prove the Jacobian conjecture one needs only to show that every map in $\mathcal{K}$ is injective, and only to prove this for cubic maps in $\mathbb{R}^{n}[11,7]$.

The structure of $\mathcal{C}$ is defined by the result of Jung:

Theorem 1 (Jung [5]). The affine Cremona group is generated by the affine, $\mathcal{A}$, and elementary, $\mathcal{E}$, subgroups.

\footnotetext{
${ }^{1}$ More generally, the term Cremona maps also refers to injective rational maps [8]. Alternative notations for $\mathcal{C}$ are $G A_{2}$ or $\operatorname{Aut}\left(\mathbf{A}^{2}\right)$.
} 
Transformations that can be written as a finite composition of elementary and affine maps are called tame; thus, Jung's theorem asserts that the planar automorphisms are tame. It is not known whether this is true in higher dimensions. A constructive proof of Jung's theorem is easily obtained based on a lemma of McKay and Wang:

Lemma 2 (McKay and Wang, [12]). Suppose $f(x, y)=(X(x, y), Y(x, y))$ is a Cremona map, and let $m=\operatorname{deg}(X(0, y))$ and $n=\operatorname{deg}(Y(0, y))$. Then

$$
\begin{aligned}
X(x, y) & =X_{m}(x) y^{m}+X_{m-1}(x) y^{m-1}+\ldots+X_{0}(x) \\
Y(x, y) & =Y_{n}(x) y^{n}+Y_{n-1}(x) y^{n-1}+\ldots+Y_{0}(x) .
\end{aligned}
$$

where $X_{j}$ and $Y_{j}$ are polynomials in $x$. Moreover, if both $m$ and $n$ are nonzero then $X_{m}$ and $Y_{n}$ are nonzero constants and either $n \mid m$ or $m \mid n$.

If we accept this nontrivial lemma, the proof of Jung's theorem is straightforward. Moreover, the proof leads to a constructive algorithm for the decomposition:

Proof. (Jung's theorem) Use the notation of Lem. 2. If $m=0$, then $X(x, y)$ is a polynomial in $x$, and so the Jacobian of $f$ is

$$
\operatorname{det} J=\frac{d X}{d x} \frac{\partial Y}{\partial y}
$$

Since det $J$ is nonzero and constant then $X(x, y)=\alpha x+\xi$ and $Y(x, y)=\beta y+p(x)$, with $\alpha \gamma \neq 0$. Thus $f$ is elementary. On the other hand if $n=0$, then $Y(x, y)$ is a polynomial in $x$, and by the same argument $f$ reduces to $(p(x)+\beta y, \gamma x+\delta)$, which is the composition of the reflection $r(x, y)=(y, x)$ with an elementary map.

So we can now assume that the degrees are nonzero, and by Lem. 2 we know that one divides the other. Take the first case, that $m \mid n$. Let $k=n / m \leq n$, and let $\lambda=Y_{n} / X_{m}^{k}$ be a constant (note: $k=1$ is permitted here). Letting $e(x, y)=\left(x, y-\lambda x^{k}\right)$ be an elementary map, then the composition

$$
\hat{f}(\hat{X}, \hat{Y})=e \circ f=\left(X(x, y), Y(x, y)-\lambda X^{k}(x, y)\right)
$$

is a polynomial automorphism such that $\operatorname{deg}(\hat{Y}(0, y))=\hat{n}<n$. The same process can be used to reduce $m$ if $n \mid m$, though we must compose with a reflection as well as an elementary map. Thus so long as $\hat{m}$ and $\hat{n}$ are nonzero, we can begin again, reducing either $\hat{m}$ or $\hat{n}$ until one of them is zero. But in this case we have already shown that the map is elementary or the composition of the reflection with an elementary map. Thus $\hat{f}$ is tame and by construction so is $f$.

Friedland and Milnor [6] have used Jung's result to obtain a dynamically useful decomposition of $\mathcal{C}$ : they show that every Cremona map is conjugate to a composition of generalized Hénon maps, or else has trivial dynamics (see Th. 4 below). Their analysis begins by noting that Th. 1 implies that any Cremona map can be written as a finite composition of elementary and affine maps. This sequence can be reduced noting that if any two consecutive factors belong to the same subgroup, they can be combined into one factor, since compositions of elementary maps are elementary. Moreover, if any factor belongs to the intersection $\mathcal{S}=\mathcal{E} \cap \mathcal{A}$, it can be composed with either factor to the right 
or the left. If those happen to be of the same type all three can be combined into one map of the same type. Thus any Cremona map $f$ can be written as a reduced map in the form

$$
f=g_{n} \circ g_{n-1} \circ \ldots \circ g_{1}
$$

such that each $g_{i}$ belongs to $\mathcal{E}$ or $\mathcal{A}$, and no two consecutive factors belong to the same subgroup.

If the length $n$ of the reduced map of $f$ is one, then it is either affine or elementary, and the dynamics is trivial. When $n>1$, the factors can always be cyclically permuted by conjugation, i.e. $f \cong g_{1} \circ f \circ g_{1}^{-1}=g_{1} \circ g_{n} \circ \ldots \circ g_{2}$. If $n$ is odd, the reduced map begins and ends with elements of the same subgroup. In this case we can permute the first element to the end and combine these two factors so that the reduced map has even length. Finally, we can cyclically permute so that $f$ is conjugate to a map that begins with an affine map:

$$
f \cong a_{k} \circ e_{k} \circ a_{k-1} \circ \ldots \circ e_{1},
$$

where the length $n=2 k$, and each factor is not in the subgroup $\mathcal{S}$. A map of the form (6) is said to be cyclically reduced.

The degree of a general Cremona map is only bounded by the product of the degrees of its factors; however, for a cyclically reduced map we have

Lemma 3 (Friedland and Milnor). The degree of a cyclically reduced map is the product of the degrees of the elementary factors.

Proof. We prove this inductively. Using (5), the degree of an elementary map $e=(X, Y)$ is $\operatorname{deg}(Y)=\operatorname{deg}(p(x))$. For the inductive step we must show that if $f=(X, Y)$ is a Cremona map and $\operatorname{deg}(f)=\operatorname{deg}(Y)=d_{0}$, then $e \circ a \circ f$ has the same property, and has the degree $\operatorname{deg}(e) \operatorname{deg}(f)$. Since our map is reduced, $a$ is an affine map that is not in $\mathcal{S}$. Using (4) this implies $L_{12} \neq 0$. Letting $f^{\prime}=\left(X^{\prime}, Y^{\prime}\right)=a \circ f$, we see that $\operatorname{deg}\left(f^{\prime}\right)=\operatorname{deg}\left(X^{\prime}\right)=d_{0} \geq \operatorname{deg}\left(Y^{\prime}\right)$. Since $e$ is an elementary map that is not in $\mathcal{S}$, $\operatorname{deg}(e)=d_{1}>1$. Using (5) we have

$$
\left(X^{\prime \prime}, Y^{\prime \prime}\right)=e \circ f^{\prime}=\left(\alpha X^{\prime}(x, y)+\xi, \beta Y^{\prime}(x, y)+p\left(X^{\prime}(x, y)\right)\right),
$$

has degree given by $\operatorname{deg}\left(Y^{\prime \prime}\right)=d_{0} d_{1}>d_{0}=\operatorname{deg}\left(X^{\prime \prime}\right)$. Thus we stay in the class of maps with $\operatorname{deg}(Y) \geq \operatorname{deg}(X)$ whenever we compose with alternating affine and elementary maps. By induction we obtain the result.

With this preparation, we can state the reduction theorem:

Theorem 4 (Friedland and Milnor). Every cyclically reduced element $f \in \mathcal{C}$ of length $2 k$ is conjugate to a composition of $k$ generalized Hénon maps, $h_{i}(x, y)=\left(y+p_{i}(x),-b_{i} x\right)$, where $p_{i}$ has degree $d_{i}$ and

$$
p_{i}(x)= \pm x^{d_{i}}+O\left(x^{d_{i}-2}\right) .
$$

If $\operatorname{deg}(f)=\prod d_{i}$ is even then all the signs above are +1 ; otherwise, at most one of the signs is negative. 
Proof. We begin with a cyclically reduced map of the form (6). First we show that the affine maps can be combined with the elementary maps to give Hénon maps. In fact each affine map can be written as $a=s^{\prime} \circ r \circ s$, where $r(x, y)=(y, x)$ is the reflection and $s, s^{\prime} \in \mathcal{S}$. To see this, note that an affine-elementary map $s \in \mathcal{S}$ has the form $s(x, y)=(\alpha x+\xi, \gamma x+\delta y+\eta)$, where $\alpha \delta \neq 0$. The composition $s^{\prime} \circ r \circ s$ gives an affine map, (4), with

$$
L=\left(\begin{array}{cc}
\alpha^{\prime} \gamma & \alpha^{\prime} \delta \\
\gamma^{\prime} \gamma+\delta^{\prime} \alpha & \gamma^{\prime} \delta
\end{array}\right)
$$

Thus the coefficients of $s$ and $s^{\prime}$ can be chosen to obtain an arbitrary element $a$, since we know that $L_{12}=\alpha^{\prime} \delta \neq 0$, or $a \notin \mathcal{S}$. Thus the cyclically reduced map can be rewritten

$$
f=s_{k}^{\prime} \circ r \circ s_{k} \circ e_{k} \circ s_{k-1}^{\prime} \ldots \circ r \circ s_{1} \circ e_{1} .
$$

Since $\mathcal{S} \subset \mathcal{E}$, the combination $s_{j} \circ e_{j} \circ s_{j-1}^{\prime}=\hat{e}_{k}$ is another elementary map, so that upon conjugation with $s_{k}^{\prime}$ we have

$$
f \cong r \circ \hat{e}_{k} \circ r \circ \hat{e}_{k-1} \ldots \circ r \circ \hat{e}_{1} .
$$

We drop the"s. Each map $r \circ e_{j}$ has the form

$$
r \circ e_{j}=\left(\beta_{j} y+p_{j}(x), \alpha_{j} x+\xi_{j}\right) .
$$

These maps can be simplified with the map $s_{j}(x, y)=\left(\alpha_{j} x+\xi_{j}, \alpha_{j+1} \beta_{j} y\right)$. Inserting factors of $s_{j}^{-1} \circ s_{j}$ and conjugating with $s_{1}$ we obtain

$$
f \cong s_{1} \circ r \circ e_{k} \circ s_{k}^{-1} \circ s_{k} \circ \ldots \circ s_{2}^{-1} \circ s_{2} \circ r \circ e_{1} \circ s_{1}^{-1} \text {. }
$$

Each combination

$$
h_{j}(x, y)=s_{j+1} \circ r \circ e_{j} \circ s_{j}^{-1}(x, y)=\left(y+q_{j}(x),-\delta_{j} x\right),
$$

is a Hénon map, where $q_{j}(y)=\alpha_{j+1} p_{j}\left(\left(x-\xi_{j}\right) / \alpha_{j}\right)+\xi_{j+1}$, and $\delta_{j}=-\alpha_{j+2} \beta_{j+1}$. Thus we see that

$$
f \cong h_{k} \circ h_{k-1} \circ \ldots \circ h_{1} .
$$

The polynomials $q_{j}$ can be normalized by a diagonal affine map. First define the translation $t_{j}=\left(x+\lambda_{j}, y-\lambda_{j-1} \delta_{j-1}\right)$, and insert factors of $t_{j}^{-1} \circ t_{j}$ as we did in (7). The form of $t_{j}$ is chosen so that it leaves the second component of the Hénon maps invariant so that $f$ is conjugate to a product of modified Hénon maps, $\hat{h}_{j}=t_{j+1} \circ h_{j} \circ t_{j}^{-1}$ with the polynomials $\hat{q}_{j}=q_{j}\left(x-\lambda_{j}\right)+\lambda_{j+1}+\lambda_{j-1} \delta_{j-1}$. We can always choose $\lambda_{j}$ so that the "center of mass" of the roots of $\hat{q}_{j}$ is zero, or equivalently the coefficient of $x^{d_{j}-1}$ vanishes. Next we insert a scaling map $d_{j}=\left(\alpha_{j} x, \alpha_{j+1} y\right)$ and again see that $f$ is conjugate to Hénon maps $\tilde{h}_{j}$ with polynomials $\tilde{q}_{j}=\alpha_{j+1} \hat{q}_{j}\left(x / \alpha_{j}\right)$, and modified Jacobians $\tilde{\delta}_{j}=\delta_{j} \alpha_{j+2} / \alpha_{j}$. We are free to choose the scaling factors $\alpha_{j}$ to normalize $\tilde{q}$. Suppose that $\hat{q}(x)=\kappa x^{d}+O\left(x^{d-2}\right)$, then we can scale all of the $\kappa$ coefficients to 1 if we can solve

$$
\alpha_{j}^{d_{j}}=\alpha_{j+1} \kappa_{j}
$$


with $\alpha_{k+1}=\alpha_{1}$. Expanding this recursion relation gives the equation

$$
\alpha_{1}^{d-1}=\kappa_{k} \kappa_{k-1}^{d_{k}} \kappa_{k-2}^{d_{k} d_{k-1}} \ldots \kappa_{1}^{d_{k} \ldots d_{2}}
$$

where $d=\prod d_{j}$ is the total degree. If $d$ is even this has a unique real solution for $\alpha_{1}$, and thus for all of the $\alpha_{j}$. If $d$ is odd, then there is a real solution only when the product on the right hand side is positive. When this is not the case, we must choose one of the leading coefficients of one of the polynomials to be -1 . With this choice there is a unique real solution for the $\alpha_{j}$.

This theorem implies that when the degree of a cyclically reduced map is prime, it must be conjugate to a single Hénon map. This was first shown by Engel [13].

Recall that the length of a map (6) is $n=2 k$ unless the map is trivial, and that it is conjugate to the composition of $k$ Hénon maps. Each Hénon map of degree $d$ contributes $d$ parameters to the final form. Since the total degree is the product of the degrees, maps with length 2 always have the largest number of parameters for fixed degree, except for degree 4 , where both possibilities have $4=2+2=2 \cdot 2$ parameters. In the next section we concentrate on the case of length 2 ; later we will be mainly interested in the cubic case.

\section{Generalized Hénon Maps}

In this section we study the dynamics of generalized Hénon maps

$$
h(x, y)=(y+p(x),-b x)
$$

where $p$ is a degree $d$ polynomial in the form $p(x)= \pm x^{d}+O\left(x^{d-2}\right)$. Recall that Th. 4 implies that the sign of the first term can always be chosen to be positive if $d$ is even, but may be negative when $d$ is odd. From our previous discussion, we know that any Keller map with prime degree smaller than 100 is conjugate to a generalized Hénon diffeomorphism. For the considerations in this section the fact that the degree is prime is not important. Therefore we now allow $p$ to have an arbitrary degree.

It is sufficient to consider the case $|b| \leq 1$, since the inverse of a generalized Hénon map with $|b|>1$ is conjugate to a generalized Hénon map with $|b|<1$ under the reflection $r(x, y)=(y, x)$ :

$$
r \circ h^{-1} \circ r=(y-p(-x / b),-x / b) .
$$

When $b= \pm 1(2)$ is reversible: it is conjugate to its inverse [14];

$$
R^{-1} \circ h \circ R=h^{-1}, \quad R(x, y)=(-y,-x) .
$$

Since $R^{-1}=R$, the reversor is an involution, and $h$ can be factored into a product of involutions, $h=R \circ(R \circ h)$. The involution $R \circ h$ is also a reversor for $h$. The fixed sets of the reversors $\operatorname{Fix}(R)=\{(x, y): y=-x\}$, and $\operatorname{Fix}(R h)=\{(x, y): 2 y=-p(x)\}$ and their iterates play an important role in finding symmetric periodic orbits of the map. 
When the polynomial $p$ is odd, the generalized Hénon map has a symmetry:

$$
S^{-1} \circ h \circ S=h, S(x, y)=(-x,-y) \text { when } p(x)=-p(-x) .
$$

For the cases $b= \pm 1$, this induces a second reversor $S \circ R$. By direct calculation one can verify that other real linear symmetries do not exist. For maps in $\mathbb{C}^{2}$, however, complex scalings can lead to symmetries; for example, the degree $d$ map with $p(x)=x^{d}+l x$ is invariant under the scaling $(x, y) \rightarrow \exp (2 \pi i /(d-1))(x, y)$.

Since area-preserving, generalized Hénon maps are reversible, they are not generic. This is somewhat surprising, because in some sense any smooth area-preserving map can be approximated by a polynomial map. However, generically an area-preserving map does not have any symmetries. We expect that polynomial maps with length greater than 2 are generically not reversible, but do not know of a proof. A polynomial area preserving map that is not reversible must have a cyclically reduced form with nonprime degree.

\subsection{Fixed Points}

A fixed point $\left(x_{*}, y_{*}\right)$ of the map satisfies $y_{*}=-b x_{*}$, and $(1+b) x_{*}=p\left(x_{*}\right)$, so that $x_{*}$ is a root of the polynomial

$$
g_{1}(x)=p(x)-(1+b) x
$$

Thus all fixed points are located on the line $y+b x=0$ in the plane - when $b=1$ this is the set $\operatorname{Fix}(R)$ - so that all fixed points are symmetric. There are at most $d$ fixed points and when $d$ is odd there always is at least one fixed point.

The stability of the fixed points is determined by the Jacobian

$$
J=\left(\begin{array}{cc}
p^{\prime}\left(x_{*}\right) & 1 \\
-b & 0
\end{array}\right)
$$

which has trace $t_{r}=p^{\prime}\left(x_{*}\right)$ and determinant $b$. The fixed point is stable if its parameters belong to the interior of the triangle $\left|t_{r}\right|-1 \leq b \leq 1$ in $\left(t_{r}, b\right)$ space, because then $J$ is a contraction. The fixed point is linearly stable on the boundary of the triangle except at the two corners $t_{r}= \pm 2$, where it is unstable. So it is necessary that $\left|t_{r}\right| \leq 1+b$ for stability. At the fixed point we have $p\left(x_{*}\right)=(1+b) x_{*}$ and $t_{r}=p^{\prime}\left(x_{*}\right)$. Dividing these two equations we find the necessary stability criteria

$$
\left|\frac{t_{r}}{1+b}\right|=\left|x_{*} \frac{p^{\prime}\left(x_{*}\right)}{p\left(x_{*}\right)}\right|=\left|\sum_{i=1}^{d} \frac{1}{1-\frac{x_{i}}{x_{*}}}\right| \leq 1,
$$

where we have assumed that $p(x)= \pm \prod_{i=1}^{d}\left(x-x_{i}\right)$, has no double roots.

Consider the " + " case, $p(x)=+x^{d}+\ldots$, and suppose $x_{*}$ is the largest of all of the fixed points. Then $x_{*}$ is necessarily unstable, because the graph of $p(x)$ crosses that of the line $(1+b) x$ from below to above at $x_{*}$ on its way to $+\infty$, so that $t_{r}=p^{\prime}\left(x_{*}\right)>(1+b)$. Therefore, if there are any fixed points, the largest one is unstable. By contrast, for the "-" case (which we only need to consider when $d$ is odd), it is possible that there is only a single, stable fixed point (see $\S 4.1)$. 


\subsection{Birkhoff Normal Form}

The stability of an elliptic fixed point of an area-preserving map is a more delicate matter than the case $|b|<1$. We investigate the neighborhood of such a fixed point by using the Birkhoff normal form. To do this we shift the fixed point $\left(x_{*},-x_{*}\right)$ to the origin, to obtain a shifted map:

$$
\left(\begin{array}{l}
x^{\prime} \\
y^{\prime}
\end{array}\right)=J\left(\begin{array}{l}
x \\
y
\end{array}\right)+\left(\begin{array}{c}
N(x) \\
0
\end{array}\right), \quad J=\left(\begin{array}{cc}
t_{r} & 1 \\
-1 & 0
\end{array}\right),
$$

where $N(x)=p\left(x+x_{*}\right)-p\left(x_{*}\right)-t_{r} x$ comprises the nonlinear terms. Suppose that $\left|t_{r}\right|<2$ so the fixed point is elliptic with rotation number $\omega$. Then $J$ has eigenvalues $\lambda=e^{2 \pi i \omega}$ and $\bar{\lambda}$ and corresponding eigenvectors $v$ and $\bar{v}$. $J$ can be diagonalized by the transformation $(x, y) \rightarrow(z, \bar{z})$ defined by $z=\bar{v} \times(x, y)$, and its complex conjugate. It is convenient to normalize the eigenvectors so that $\bar{v} \times v=2 i$ for then $z=\sqrt{2 r} e^{i \theta}$, where $(\theta, r)$ are canonical angle-action variables. This defines $v$ up to a scaling of modulus one, which we choose so that the expression for $x$ is simple, since the nonlinearity is purely in $x$. This is achieved with the choice $v=(i,-i \bar{\lambda}) / w$ where $w=\sqrt{\sin 2 \pi \omega}$ so that $x=\frac{z+\bar{z}}{2 w}$. In these complex coordinates the map becomes

$$
z^{\prime}=\lambda(z-i Q(z+\bar{z}))
$$

where $Q(\zeta)=\frac{1}{w} N(\zeta /(2 w))$. By Taylor expansion of $p\left(x+x_{*}\right)$ we obtain

$$
Q(\zeta)=\frac{\alpha}{2} \zeta^{2}+\frac{\beta}{3} \zeta^{3}+\frac{\gamma}{4} \zeta^{4} \ldots,
$$

where the first three coefficients of $Q$ are given by

$$
\alpha=\frac{p^{\prime \prime}\left(x_{*}\right)}{4 w^{3}}, \quad \beta=\frac{p^{\prime \prime \prime}\left(x_{*}\right)}{16 w^{4}}, \quad \gamma=\frac{p^{(4)}\left(x_{*}\right)}{96 w^{5}} .
$$

For the quadratic map, $\gamma=\beta=0$, and $\alpha$ is independent of the position of the fixed point $x_{*}$. For the cubic map $\gamma=0, \beta$ is independent of $x_{*}$, and only $\alpha$ depends on $x_{*}$.

When the map has no low-order resonances $\left(\lambda^{k} \neq 1\right.$ for $\left.k \leq 6\right)$ we can perform a coordinate transformation to reduce it to the Birkhoff normal form

$$
\begin{aligned}
& r^{\prime}=r+O\left(r^{3 / 2}\right), \\
& \theta^{\prime}=\theta+2 \pi\left(\omega+\tau_{0} r+\frac{1}{2} \tau_{1} r^{2}\right)+O\left(r^{5 / 2}\right),
\end{aligned}
$$

where $\tau_{0}$ and $\tau_{1}$ are the first two "twist" coefficients of the taylor series of the twist $\partial \theta^{\prime} / \partial r$. The first twist, $\tau_{0}$, is defined when $\omega \neq 0,1 / 2$, or $1 / 3$, and $\tau_{1}$ is defined if in addition $\omega \neq 1 / 4,1 / 5$, or $2 / 5$. After some calculations (see e.g., [15]) we find

$$
\pi \tau_{0}=-\beta-\frac{3 t^{2}-5}{t\left(t^{2}-3\right)} \frac{\alpha^{2}}{2}, \quad t \equiv \tan (\pi \omega) .
$$

Note that whenever $\alpha \neq 0, \tau_{0}$ approaches infinity when $\omega \rightarrow 0, \frac{1}{2}$ or $\frac{1}{3}$, where the Birkhoff transformation is not defined. These correspond to saddle-node, period doubling bifurcations, and tripling bifurcations, respectively. 
As is well known, the Moser twist theorem [16] implies that the elliptic fixed point is stable when $\tau_{0} \neq 0$, and $\omega \neq 0,1 / 2,1 / 3$ or $1 / 4$. Moreover, when the first twist vanishes at an elliptic fixed point, this point may be unstable, but then $\tau_{1} \neq 0$ is sufficient for stability if there is no low-order resonance $[16,17]$. For generalized Hénon maps the second twist is given by

$$
\pi \tau_{1}=\pi \tau_{0} \tau_{01}+\tau_{11}
$$

where

$$
\begin{aligned}
\tau_{01} & =-\frac{\alpha^{2}}{12} \frac{1095-2528 t^{2}+2366 t^{4}-872 t^{6}+51 t^{8}}{t^{2}\left(t^{2}-3\right)\left(t^{2}-1\right)}-\frac{\beta}{6} \frac{17-38 t^{2}+17 t^{4}}{t\left(t^{2}-1\right)}, \\
\tau_{11} & =\frac{4 \alpha^{4}}{3} \frac{105-305 t^{2}+353 t^{4}-167 t^{6}+30 t^{8}}{\left(t^{2}-1\right)\left(t^{2}-3\right)^{3} t^{3}}-6 \alpha \gamma \frac{5 t^{2}-7}{t\left(t^{2}-3\right)} .
\end{aligned}
$$

Thus when $\tau_{0}=0$, we have $\pi \tau_{1}=\tau_{11}$. In the special case $\gamma=0$ (as for the cubic map) the second term vanishes, and since the polynomial in the numerator of the remaining term of $\tau_{11}$ does not have real roots, $\tau_{1}$ never vanishes when $\tau_{0}$ does. Thus if $\alpha \neq 0$, we see that $\tau_{1} \neq 0$ when $\tau_{0}=0$, and the fixed point is stable unless there is a low-order resonance. We will discuss the low-order resonant cases for the cubic map in more detail in $\S 4$.

\subsection{Periodic Orbits}

In principle, periodic orbits are given by the equation $h^{n}(x, y)=(x, y)$ which is equivalent to two polynomials, one of degree $d^{n}$ and the other of degree $d^{n-1}$. However, these degrees are not optimal; to obtain polynomials of minimal degree, we make use of the fact that the inverse of $h$ also is a polynomial equation. For example, for period 2 orbits we write $h(x, y)=h^{-1}(x, y)$, and obtain the system $\left(1+\frac{1}{b}\right) y+p(x)=0$ and $(1+b) x-p(-y / b)=0$, hence the period two orbits are roots of

$$
g_{2}(x)=(1+b) x-p\left(\frac{p(x)}{1+b}\right)
$$

The fixed points are also roots of this equation, thus $g_{2}$ is divisible by $g_{1}(x)=p(x)-$ $(1+b) x$. Thus there are at most $d^{2}-d$ period points of minimal period 2 , and therefore $\left(d^{2}-d\right) / 2$ period 2 orbits.

The equations for periodic orbits of higher period can not easily be reduced to one polynomial equation. In the general case we can reduce to a system of two equations. For example, period 3 orbits are determined by the system

$$
x_{0}+b x_{1}=p\left(p\left(x_{1}\right)-b x_{0}\right), \quad x_{1}-b^{2} x_{0}=p\left(x_{0}\right)-b p\left(x_{1}\right) .
$$

Similarly for period 4 we obtain the system of equations

$$
\begin{aligned}
& x_{1}(b-1 / b)+p\left(x_{0}\right)=p\left(p\left(x_{1}\right)-b x_{0}\right), \\
& x_{0}\left(1-b^{2}\right)+b p\left(x_{1}\right)=p\left(p\left(x_{0}\right) / b-x_{1} / b\right) .
\end{aligned}
$$


In general we can easily reduce the period $n$ case to two polynomials of degree $d^{n / 2}$ when $n$ is even or of degrees $d^{(n-1) / 2}$ and $d^{(n+1) / 2}$ when $n$ is odd. These equations are defined by the recursion

$$
F_{i}=-b F_{i-2}+p\left(F_{i-1}\right), \quad F_{0}=x_{0}, \quad F_{1}=x_{1},
$$

and a similar recursion working backwards

$$
b G_{i}=-G_{i+2}+p\left(G_{i+1}\right), \quad G_{n}=x_{0}, \quad G_{n+1}=x_{1} .
$$

Of course these recursions give $F_{t}=G_{t}=x_{t}$ if we apply them for $n$ steps. To minimize the degrees of the polynomials however, we apply them only for half of the period. For example, when $n$ is even, we can apply both recursions for $n / 2$ steps, giving the two equations

$$
G_{n / 2+1}+b F_{n / 2-1}=p\left(F_{n / 2}\right), \quad G_{n / 2+2}+b F_{n / 2}=p\left(G_{n / 2+1}\right) .
$$

In this way the periodic orbits of period $n$ are determined by two equations of degree $d^{n / 2}$, so that by Bezout's theorem the maximum number of solutions is $d^{n}$. Note here that the use of the standard version of Bezout's Theorem is sufficient because we kept the degree to a minimum, while in [6] a more complicated version was needed. This result suggests that $d$ symbols should be enough to code all periodic orbits (see $\S 3.4$ below). It also shows that the growth rate of the number of periodic points is bounded above by $\ln d$. The topological entropy is also bounded by this quantity [6].

Linear stability of periodic orbits is determined by the trace and determinant of $D h^{n}$. In general, the determinant is $b^{n}$. The trace is more complicated, for example for periods 2 and 3 we obtain

$$
\begin{aligned}
& t_{r 2}=p^{\prime}\left(x_{0}\right) p^{\prime}\left(x_{1}\right)-2 b, \\
& t_{r 3}=p^{\prime}\left(x_{0}\right) p^{\prime}\left(x_{1}\right) p^{\prime}\left(x_{2}\right)-b\left(p^{\prime}\left(x_{0}\right)+p^{\prime}\left(x_{1}\right)+p^{\prime}\left(x_{2}\right)\right) .
\end{aligned}
$$

For the area-preserving case some simplification can be obtained if we restrict to the case of symmetric orbits. Symmetric periodic orbits have two points on $\operatorname{Fix}(R) \cup \operatorname{Fix}(R h)$ [14]. This means we can eliminate $y$, and treat a single equation in $x$. Again polynomials of the lowest degree can be obtained by using equal numbers of compositions of $h$ and its inverse. It is interesting that fixed points always fall on the intersection of the two fixed sets, $\operatorname{Fix}(R) \cap \operatorname{Fix}(R h)$. Period 2 orbits satisfy the equations $2 y_{t}=-p\left(x_{t}\right)$ and so must lie on $\operatorname{Fix}(R h)$. Orbits of higher period, however, need not be symmetric, see $\S 4.3$.

\subsection{Anti-Integrable Limit}

A generalized Hénon map can be obtained from the Lagrangian generating function [18]

$$
L\left(x, x^{\prime}\right)=-m x x^{\prime}+U(x),
$$

where the potential $U(x)$ is a degree $d+1$ polynomial. Here we have inserted an additional parameter, the "mass" $m$, for convenience. The equations of motion are determined by $d L=b^{-1} y^{\prime} d x^{\prime}-y d x$ or equivalently

$$
\left(x^{\prime}, y^{\prime}\right)=\left(\frac{1}{m}\left(y+U^{\prime}(x)\right),-m b x\right) \text {. }
$$


The map (19) is equivalent to the generalized Hénon map (2) if we conjugate with the scaling map $(x, y) \rightarrow(s x, s y / m)$ and let $U^{\prime}(x)=\frac{m}{s} p(s x)$, where $s=m^{\frac{1}{1-d}}$.

Alternately, the dynamics takes place on the space of sequences $\left\{x_{t}, t \in \mathbb{Z}\right\}$, and orbits are critical points of the formal action sum $\sum_{t} b^{-t} L\left(x_{t}, x_{t+1}\right)$. The Euler-Lagrange equation for this is

$$
m\left(x_{t+1}+b x_{t-1}\right)=U^{\prime}\left(x_{t}\right) .
$$

The anti-integrable limit of this map corresponds to $m=0$, where the action reduces to $\sum_{t} b^{-t} U\left(x_{t}\right)$. Thus in this limit the "orbits" of the system correspond to arbitrary sequences of the critical points of $U(x)$ [19]. If $U$ has $j \leq d$ critical points, then the "dynamics" in this limit is simply the full shift on $j$ symbols. The anti-integrable limit is called nondegenerate if the critical points of $U$ are nondegenerate. In this case there is an $\epsilon>0$, such that every sequence of critical points continues to a unique orbit of the map (20) providing $|m|<\epsilon$. Thus for small enough $|m|$ the bounded orbits of (20) can be put in one-to-one correspondence to a full shift on $j$ symbols.

It is not difficult to obtain explicit estimates for $\epsilon$ :

Theorem 5 (Anti-Integrable Continuation [20]). Let $\left\{z_{i}, i=1 \ldots j\right\}$ be the set of nondegenerate critical points of a fixed $C^{2}$ function $U$, and suppose $Z=\max _{i}\left(\left|z_{i}\right|\right)$. Choose values $\alpha>0$ and $\delta>0$ so that

$$
\left|U^{\prime \prime}(x)\right|>\alpha \quad \text { when } \quad\left|x-z_{i}\right|<\delta .
$$

Then every sequence of critical points continues to a unique, hyperbolic orbit of the map (20) providing

$$
|m|(1+|b|)<\frac{\alpha \delta}{\delta+Z} .
$$

Proof. We sketch the proof. Suppose $x_{t}(0)=z_{i(t)}$ is a sequence of critical points for any sequence $\{i(t): t \in \mathbb{Z}, 1 \leq i(t) \leq j\}$. Our goal is to find a continuation of this orbit, $x_{t}(m)$, for $m \neq 0$. Let $\xi_{t}(m)=x_{t}(m)-z_{i(t)}$, and differentiate (20) with respect to $m$ to obtain a set of differential equations for $\xi_{t}$ :

$$
\left(U^{\prime \prime}\left(x_{i}\right) \delta_{i, j}-m G_{i, j}\right) \frac{d \xi_{j}}{d m}=G_{i, j} x_{j}(m)
$$

where $G_{i, j}=\delta_{i, j}+b \delta_{i-1, j}$. The initial value problem, $\xi_{i}(0)=0$, has a unique solution since $\left|U^{\prime \prime}\left(z_{i}\right)\right|>\alpha$ and $\| G||=1+|b|$ imply that the operator on the left side of the equation is invertible when $m(1+|b|)<\alpha$. Using the assumed bounds on $U^{\prime \prime}$ it is easy to bound the solution by

$$
\left|\xi_{t}\right|<Z \frac{m(1+|b|)}{\alpha-m(1+|b|)} .
$$

Thus we can guarantee that $\left|\xi_{t}\right|<\delta$ when $m$ satisfies (22).

For the quadratic Hénon map, a better bound was obtained in [21]. We will translate (22) into an explicit form in $\S 4.5$ for the cubic case. 


\subsection{Bounded Orbits}

It is easy to see that any generalized Hénon map can have bounded orbits only when there are fixed points:

Theorem 6 (Unbounded Orbits [6]). Suppose a generalized Hénon map (2) has no fixed points. Then every orbit is unbounded.

Proof. Let $h(x, y)=(y+p(x),-b x)$. Suppose that there are no fixed points. Then the polynomial $g_{1}=p(x)-(1+b) x$ is either positive or negative for all $x$. Now consider the function $d(x, y)=x+y$. Note that

$$
d^{\prime}=d\left(x^{\prime}, y^{\prime}\right)=y+p(x)-b x=d(x, y)+g_{1}
$$

If $g_{1}>0$, then $d$ grows monotonically and must be unbounded. If $g_{1}<0$, then $d$ grows monotonically in backwards time. In either case there are no orbits bounded for all time, since then $d$ would have to be bounded.

In a more general context, it is known that any homeomorphism of the plane that has a periodic point must have a fixed point [22]. It is not clear, however, that any homeomorphism with a bounded orbit necessarily has a periodic orbit.

When there are fixed points, we can find a box that contains all of the bounded orbits:

Theorem 7 (Bounded Orbits). Every bounded orbit of a generalized Hénon map (2) is contained in the box

$$
\{(x, y):|x| \leq M,|y| \leq|b| M\}
$$

where $M$ is the largest of the absolute values of the roots of $|p(x)|-(1+|b|)|x|$.

Proof. Write the map in second difference form $x_{t+1}+b x_{t-1}=p\left(x_{t}\right)$. Since the degree $d>1, p$ grows superlinearly, and there is a constant $M>0$ such that if $|x|>M$, then $|p(x)|>(1+|b|)|x|$. Assuming $\left|x_{0}\right|>M$, we have

$$
\left|x_{1}\right|+|b|\left|x_{-1}\right| \geq\left|p\left(x_{0}\right)\right|>(1+|b|)\left|x_{0}\right|
$$

so that either $\left|x_{1}\right|>\left|x_{0}\right|$, or $|b|\left|x_{-1}\right|>|b|\left|x_{0}\right|$. In the first case we can see that $\left|x_{t}\right|$ is unbounded since the next iterate gives

$$
\left|x_{2}\right|+|b|\left|x_{0}\right| \geq\left|p\left(x_{1}\right)\right|>(1+|b|)\left|x_{1}\right|
$$

so that $\left|x_{2}\right|>(1+|b|)\left|x_{1}\right|-|b|\left|x_{0}\right|>\left|x_{1}\right|$. Thus $\left|x_{t}\right|$ forms an increasing sequence as $t$ increases. This sequence is unbounded since any limit point would have to be a solution of $|x|(1+|b|)=|p(x)|$, but $|x|>M$, so there is no such solution. A similar analysis applies to the second case to show that $\left|x_{t}\right|$ is unbounded as $t$ decreases.

For example, for the quadratic Hénon map, $p(x)=x^{2}+a$, Th. 6 implies that there can be bounded orbits only when $\Delta=(1+b)^{2} / 4-a>0$. In this case Th. 7 gives $M=\frac{1+|b|}{2}+\sqrt{\frac{(1+|b|)^{2}}{4}-a}$. This is the position of the saddle fixed point when $b>0$.

More generally, the polynomial determining $M$ is the same as that for the fixed points, up to the absolute value signs. When $b>0$, the signs could conspire so that $M$ is in fact a fixed point. In this case, it is the largest one, and by our previous considerations in $\S 3.1$ it is an unstable fixed point. Thus in this case the box containing the bounded orbits is defined by the corner containing the largest, unstable fixed point. 


\section{Area-Preserving Cubic Diffeomorphisms}

In this section we concentrate on the area-preserving cubic maps

$$
C_{ \pm}(x, y)=\left(y \pm x^{3}+l x+k,-x\right) .
$$

We label the two cases by the sign of the cubic term. The maps $C_{ \pm}$are reversible, but have the spatial symmetry (9) only when $k=0$. Throughout this section the \pm refers to the two cases.

\subsection{Fixed Points}

A fixed point $\left(x_{*}, y_{*}\right)$ of the map satisfies $y_{*}=-x_{*}$, and $2 x_{*}=p\left(x_{*}\right)$, so that $x_{*}$ is a root of the polynomial

$$
g_{1}(x)=p(x)-2 x=k+x(l-2) \pm x^{3} .
$$

The cubic map always has at least one real fixed point, since a cubic polynomial always has one root. The explicit expressions for the fixed points are not particularly useful in general; a simple case corresponds to $k=0$, when the three fixed points are $x_{*}=$ $0, \sqrt{ \pm(2-l)},-\sqrt{ \pm(2-l)}$.

We can obtain expressions for saddle-node and period doubling bifurcation curves. Stability of a fixed point is determined by the Jacobian (10), which has the trace

$$
t_{r}=l \pm 3 x_{*}^{2}=g_{1}^{\prime}\left(x_{*}\right)+2 .
$$

Eliminating $x_{*}$ from the equation for the trace using the fixed point equation $g_{1}=0$ we obtain the resultant polynomial for $t_{r}$ depending on $k$ and $l$,

$$
\Delta_{1}\left(t_{r}\right)=-27 k^{2} \pm\left(t_{r}-l\right)\left(2 l-6+t_{r}\right)^{2} .
$$

Zeros of $\Delta_{1}\left(t_{r}\right)$ correspond to curves in the parameter space for which a fixed point has the given trace. The saddle node $(S N)$ curve corresponds to $\lambda=\bar{\lambda}=1$, so that $t_{r}=2$. For this case the resultant of $g_{1}$ and $g_{1}^{\prime}+2-t_{r}$ reduces to the discriminant of $g_{1}$,

$$
\Delta_{1}(2)=-27 k^{2} \pm 4(2-l)^{3}=0,
$$

and therefore corresponds to double roots in $g_{1}$. This curve is a semicubical or "Neils parabola;" it is the cusped curves in Fig. 1 and Fig. 2. There are three fixed points when $\Delta_{1}(2)>0$, and only one when $\Delta_{1}(2)<0$.

A period doubling bifurcation $(P D)$ occurs when $t_{r}=-2$, corresponding to the zero set of

$$
\Delta_{1}(-2)=-27 k^{2} \mp 4(l+2)(l-4)^{2} .
$$

With parameters on $\Delta_{1}\left(t_{r}\right)=0$ we do not in general have a double root, but we still can obtain a simple explicit solution for the three fixed points because we know one root of the cubic $g_{1}$ explicitly. The three roots are

$$
\begin{aligned}
x_{*}=\mu & -\frac{\nu+\mu}{2}, \frac{\nu-\mu}{2} ; \\
\mu & =\sqrt{\mp \frac{\left(l-t_{r}\right)}{3}}, \quad \nu=\sqrt{\mp\left(3 l+t_{r}-8\right)},
\end{aligned}
$$




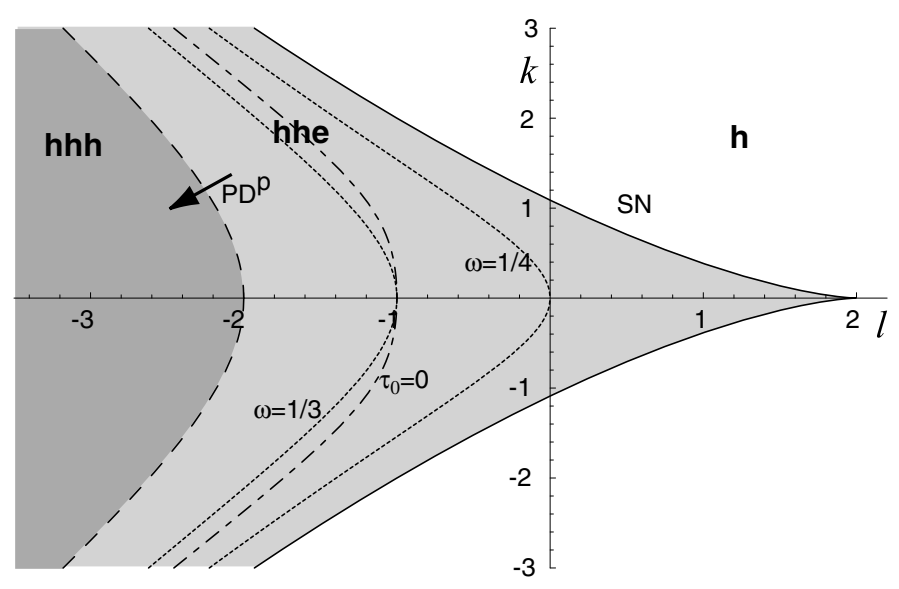

Figure 1: Bifurcation diagram for fixed points of $C_{+}$. The fixed points are denoted $\mathbf{e}$ for elliptic and $\mathbf{h}$ for hyperbolic. There are 3 fixed points in the shaded region. As in all bifurcation diagrams we use the following coding: solid for $t_{r}=2$, dashed for $t_{r}=-2$, dotted for $t_{r}=-1,0$, long/short dashed for vanishing twist of the fixed point, $\tau_{0}=0$.

corresponding to $k>0$; the signs of $\mu$ and $\nu$ are reversed for $k<0$. The fixed point at $\mu$ is the one that has the specified trace. When $t_{r}=2$ the first and last root coincide. Even for arbitrarily large values of $(l, k)$ there are elliptic fixed points with $\left|t_{r}\right|<2$, providing $27 k^{2} \sim \mp 4 l^{3}$.

The two curves $\Delta_{1}( \pm 2)=0$ in the $(l, k)$ space constitute the bifurcation diagram for the fixed points. Since we can solve $\Delta_{1}\left(t_{r}\right)$ for $k^{2}$ for every value of $t_{r}$, we can easily calculate the lines in parameter space where the fixed point has a given multiplier. For example the rotation number is $\frac{1}{3}$ when $t_{r}=-1$, so the curve $\Delta_{1}(-1)=0$ is the tripling curve, see Fig. 1 and Fig. 2.

The structures of the two cases is rather different. For $C_{+}$one unstable fixed point always exists. A new pair of fixed points is born in a saddle node bifurcation upon crossing $\Delta_{1}(2)=0$ with decreasing $l$, see Fig. 1 The stable fixed point remains linearly stable until it crosses $\Delta_{1}(-2)=0$, where it period doubles and becomes inverse hyperbolic. For $C_{+}$ there is at most one stable fixed point.

For the map $C_{-}$we get a more fascinating scenario. The saddle node curve creates a pair of orbits when traversed in the direction of increasing $l$, see Fig. 2. But the single orbit that always exists can be stable. We have regions where there is one stable, Fig. 3, or one unstable orbit; when there are three orbits then at most two of them can be stable, see Fig. 4.

The parameter spaces of both $C_{ \pm}$have a singularity at $(l, k)=(2,0)$, where the three fixed points coincide at $(x, y)=(0,0)$. A periodic orbit continued on a closed loop in parameter space around this point might not return to the same point. In particular for $C_{-}$, if we start in the region with three fixed points, and continue the stable fixed point with initially positive $x_{*}$ around the singularity with counterclockwise orientation, we end up with the stable fixed point with negative $x_{*}$ after returning to the initial parameters.

This phenomenon is the same as that observed upon continuing the roots of the cubic polynomial $a+b x \pm x^{3}$ around a circle around the origin in parameter space $(a, b)$. In 


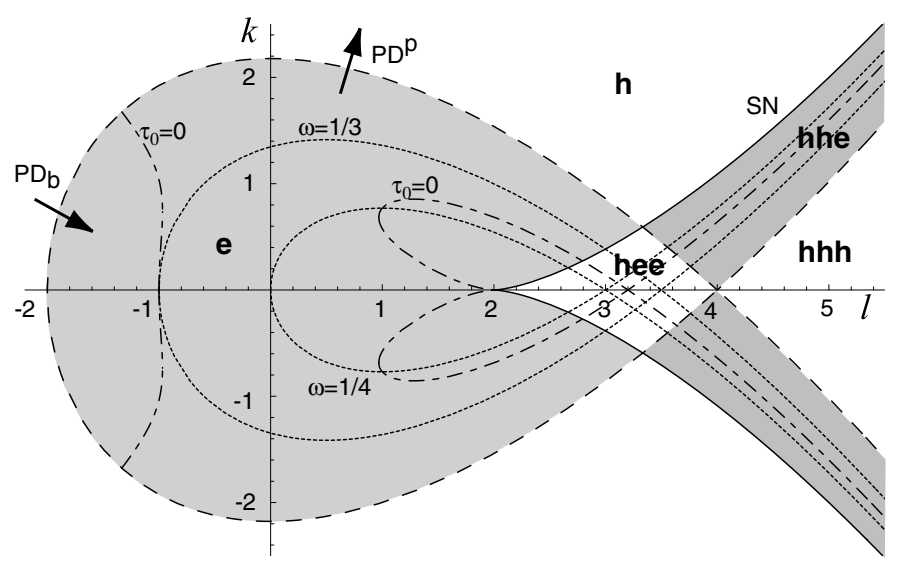

Figure 2: Bifurcation diagram for fixed points of $C_{-}$. The notation is the same as that in Fig. 1. The period doubling is subcritical $\left(P D_{b}\right)$ for $l<4 / 3$ and supercritical $\left(P D^{p}\right)$ for $l>4 / 3$, creating a period two orbit in the direction of the arrows.

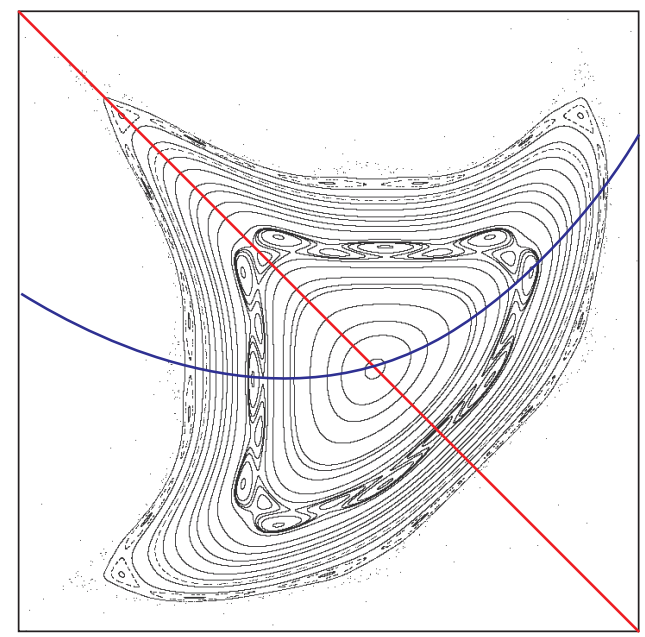

Figure 3: Phase space of $C_{-}$for $(l, k)=(1.2,1.07815)$, where there is a single elliptic fixed point. Shown are a pair of $\frac{3}{10}$ islands with a meandering "twistless" curve. Also shown are the symmetry lines $\operatorname{Fix}(R)$ and $\operatorname{Fix}(R h)$. Window bounds are $(0.2,-1.2) \times(1.2,-0.2)$. 


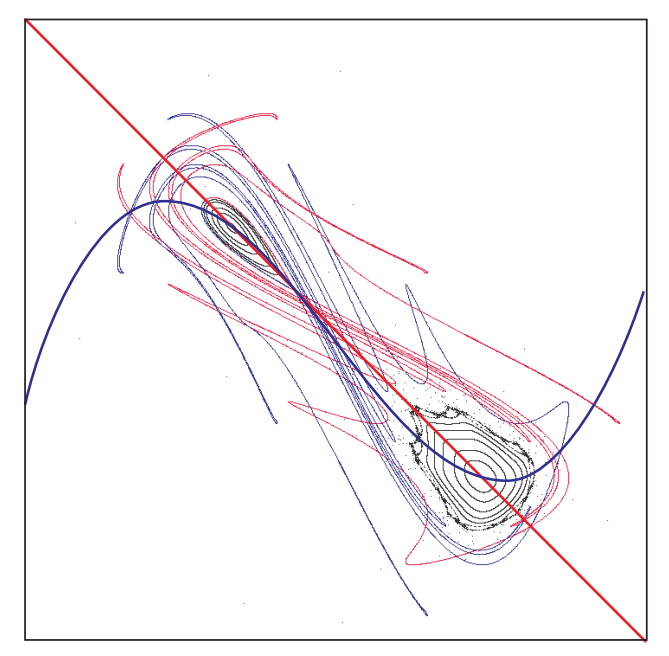

Figure 4: Phase space of $C_{-}$for $(l, k)=(2.5,0.1)$, where there are three fixed points, and two are stable. Also shown are the symmetry lines $\operatorname{Fix}(R)$ and $\operatorname{Fix}(R h)$, and the stable and unstable manifolds of the saddle fixed point. Window bounds are $(-1.7,1.7) \times(-1.7,1.7)$.

general this "hysteresis" will occur for any loop enclosing a triple zero-it is a simple example of the so-called cusp catastrophe.

\subsection{Birkhoff Normal Form}

We now investigate the behavior of $C_{ \pm}$in the neighborhood of an elliptic fixed point by using the Birkhoff normal form. The coefficients in the normal form (13) are

$$
\alpha= \pm \frac{3}{2 w^{3}} x_{*}, \quad \beta= \pm \frac{3}{8 w^{4}}, \quad \gamma=0
$$

These expressions can be substituted into the twist (14) to obtain explicit expressions for $\tau_{0}$. These are especially simple when $k=0$. The fixed point at $x_{*}=0$ has $t_{r}=l$, and so is elliptic for $|l|<2$. The twist for this case is $\pi \tau_{0}= \pm \frac{3}{2\left(l^{2}-4\right)}$, which is always nonzero. The result is simple in this case because the coefficient $\alpha$ of the quadratic term in the normal form is zero, so no transformation is needed at all to calculate the twist. In addition when $k=0$ the map $C_{-}$has an elliptic fixed point for $2 \leq l \leq 4$ with $x_{*} \neq 0$; it has twist

$$
\pi \tau_{0}=\frac{3(5 l-16)}{4(2 l-7)(l-2)(l-4)} .
$$

For this case the twist vanishes when $l=16 / 5$, which is near the third order resonance $(l=7 / 2)$. This is to be expected from the generic situation [15]. Note that this expression is valid for both elliptic fixed points, because for $k=0$ the map has the additional symmetry $S$.

In general when twist vanishes at the fixed point, $\tau_{0}=0$, we say the map has a "twistless" bifurcation [15]. The corresponding curve in parameter space can be obtained 


\begin{tabular}{c|c|c|c}
$\omega$ & $l$ & $k$ & event \\
\hline 0 & 2 & 0 & pitchfork, $\tau_{0}$ singular \\
$\frac{1}{2}$ & $-\frac{4}{3}$ & $\pm \frac{32}{27} \sqrt{2}$ & degenerate PD, unstable, $\tau_{0}$ singular \\
$\frac{1}{3}$ & -1 & 0 & symmetric $1 / 3$, stable, $\tau_{0} \neq 0$ singular \\
$\frac{1}{4}$ & 1 & $\pm \frac{4}{9} \sqrt{3}$ & $\tau_{0}=0$, stable \\
$\frac{1}{4}$ & $\frac{1}{3}$ & $\pm \frac{16}{27}$ & $\left|a_{21}\right|=\left|a_{03}\right|$, stability open \\
$\frac{1}{5}$ & $\frac{\sqrt{5}}{2}$ & $\pm \frac{3 \sqrt{30}-13 \sqrt{6}}{36}$ & $\tau_{0}=0$, unstable \\
$\frac{2}{5}$ & $-\frac{\sqrt{5}}{2}$ & $\pm \frac{3 \sqrt{30}+13 \sqrt{6}}{36}$ & $\tau_{0}=0$, unstable \\
$\frac{1}{6}$ & $\frac{4}{3}$ & $\pm \frac{7}{27}$ & $\tau_{0}=0$, stable
\end{tabular}

Table 1: Parameter values for codimension two bifurcations of the fixed point of $C_{-}$.

by eliminating $x_{*}$ from the expression for $\tau_{0}$, which gives the resultant polynomial

$$
\begin{gathered}
Z(k, l)=729 k^{4} \pm\left(8 l^{3}-108 l^{2}-63 l+837\right) k^{2} \\
-\frac{16}{27}(l+1)(5 l-16)^{2}(l-2)^{3} .
\end{gathered}
$$

For $C_{+}$, the twistless curve, $Z=0$ is very close to the curve $\Delta_{1}(-1)=0$ where the rotation number is $\frac{1}{3}$, and touches this curve at $k=0$, as can be seen in Fig. 1. For the map $C_{-}$, the corresponding curve consists of two disjoint parts. The finite piece is tangent to the period tripling curve at $(l, k)=(-1,0)$ and ends on the doubling curve. There is also an infinite piece which is asymptotic to the period tripling curve at infinity, see Fig. 2. In this case every rotation number is intersected by the twistless curve. For reference we list the intersections of $Z=0$ with low-order resonances in Table 1 . The consequences of the twistless bifurcation include reconnection bifurcations of island chains, and "meandering" invariant curves [15]; an example is shown in Fig. 3. Note that the twist $\tau_{0}$ is not a function of $k$ and $l$, because it also depends on the elliptic fixed point for which it is evaluated. This is the reason why the curve $Z=0$ intersects itself in the region where there are two elliptic fixed points. One could turn $\tau_{0}$ into a well defined function by considering it on the cusp-catastrophe surface in $\mathbb{R}^{3}$ of $k, l$ and $x_{*}$.

Now we consider the behavior near the points where $\tau_{0}$ is zero or singular. Singularities in $\tau_{0}$ occur when $\omega=0,1 / 2$ and $1 / 3$, and also at the points $(l, k)=(2,0)$, $(-4 / 3, \pm 32 \sqrt{2} / 27)$ and $(-1,0)$. For example, the point $(-1,0)$ is singular because the limit of $\tau_{0}$ is zero if we approach the point on the curve $Z=0$, but $\tau_{0}$ is infinite if we approach the point on the curve $\omega=1 / 3$. However, the value at $(-1,0)$ is just $\mp \frac{1}{2}$.

When $\tau_{0}=0$, the elliptic point is stable if it is not low-order resonant $\left(\lambda^{k} \neq 1\right.$ for $k \leq 6)$ and the second twist $\tau_{1}$ is nonzero $[16,17]$. As we noted in $\S 3.2$, when $\tau_{0}=0$, $\tau_{1}$ never vanishes when $\alpha \neq 0$. This implies that the fixed points in the families $C_{ \pm}$are stable even for vanishing twist as long as they are not low-order resonant and $\alpha \neq 0$. If $\alpha=0$ then $x_{*}=0$ but in this case the first twist is just given by $-\beta$ which is always nonzero so that we again have stability.

The stability of the fixed point for the low-order resonant singular and twistless cases is more delicate. For $\omega=1 / 2$ the fixed point is unstable because it is linearly unstable. For $C_{-}$the points $(l, k)=\left(-\frac{4}{3}, \pm \frac{4}{9} \sqrt{3}\right)$ correspond to the intersection of the twistless curve with the period doubling curve, and signal the codimension-two transition from 
supercritical to subcritical period doubling, see $\S 4.3$. When $\omega=1 / 3$ the twist is singular and the fixed point is unstable, except at the point $(l, k)=(-1,0)$ where the map has the spatial symmetry $S,(9)$. The additional symmetry implies that the resonant term vanishes, and since $\pi \tau_{0}=1 / 2 \neq 0$ at this point, the fixed point is stable [23, 24].

Since for $C_{+}$the twistless curve does not cross the low-order resonance curves $\omega=$ $1 / 4,1 / 5,2 / 5,1 / 6$ additional codimension two cases do not appear in this family. For $C_{-}$, however, there are twistless, codimension two bifurcations; these are listed in Table 1.

For $\omega=1 / 4,1 / 5$, and $2 / 5$ the normal form is

$$
z^{\prime}=\lambda\left(z+a_{2,1} z^{2} \bar{z}+a_{0, n-1} \bar{z}^{n-1}+\ldots\right)
$$

where $\lambda^{n}=1$, and $a_{2,1}=i \pi \tau_{0}$. The map is unstable when $\tau_{0}=0$, providing $a_{0, n-1} \neq 0$ $[15,24]$. For the cases in Table 1 with $\tau_{0}=0$ we find: when $\omega=1 / 4 a_{0,3}=-i 4 / 3$, and when $\omega=1 / 5,2 / 5$ we find $a_{0,4}=-(5 \sqrt{5} \pm 13)(5 \mp 2 \sqrt{5})^{3 / 4} \sqrt{3} / 80$, respectively. Thus these cases are unstable, see e.g. Fig. 5 . For $\omega=1 / 5$ the absolute value $\left|a_{0,4}\right|$ is larger than for $\omega=2 / 5$, so that the instability is more pronounced.

The second twist $\tau_{1}$ is also not defined on the entire curve $\omega=1 / 4$, i.e, $\Delta_{1}(0)=0$, recall Fig. 2. For this case, the normal form (26) applies, and the fixed point is unstable if $\left|a_{2,1}\right|<\left|a_{0,3}\right|[15]$, and stable if the inequality is reversed according to KAM theory [24]. We find that $a_{2,1}= \pm 3 i(1-l) / 8$ and $a_{0,3}= \pm i(1+3 l) / 8$, so that the fixed point of $C_{-}$is unstable for $l>1 / 3$ and stable when $0 \leq l<1 / 3$. The only case that is not decided is the point $l=1 / 3$. For $C_{+}$the fixed point with $\omega=1 / 4$ is always stable because it only exists for $l \leq 0$.

The normal form when $\tau_{0}=0$ for $\omega=1 / 6$, is

$$
z^{\prime}=\lambda\left(z+a_{3,2} z^{3} \bar{z}^{2}+a_{0,5} \bar{z}^{n-1}+\ldots\right)
$$

where $a_{3,2}=i \pi \tau_{1} / 4$. The fixed point is stable if $\left|a_{32}\right|>\left|a_{05}\right|$ [24]. At the point in Table 1 where $\omega=1 / 6$, we find $a_{3,2}=i 31 \sqrt{3} / 72$ while the resonant term $a_{0,5}=i 13 \sqrt{3} / 72$ is much smaller; thus this point is stable [24].

We summarize the stability results of this section in the following

Theorem 8. The elliptic fixed point of the cubic area preserving map $C_{+}$is unstable when $\omega=1 / 3$ and $k \neq 0$ but is otherwise stable. An elliptic fixed point of the cubic area preserving map $C_{-}$is unstable for the cases $\omega=1 / 3$ and $k \neq 0, \omega=1 / 4$ and $l>1 / 3$, $\omega=1 / 5$ and $l=\sqrt{5} / 2$, and when $\omega=2 / 5$ and $l=-\sqrt{5} / 2$. It is stable in all other cases except possibly for $\omega=1 / 4$ and $l=1 / 3$.

\subsection{Periodic Orbits for $C_{-}$}

We next study some of the low period periodic orbits of $C_{-}$. First recall that the period two orbits of a generalized, area-preserving Hénon map are always located on the symmetry line $\operatorname{Fix}(R h)$, i.e. $2 y=-p(x)$, and are determined by (15), which is equivalent to

$$
g_{2}(x)=2 p(p(x) / 2)-4 x=0 \text {. }
$$




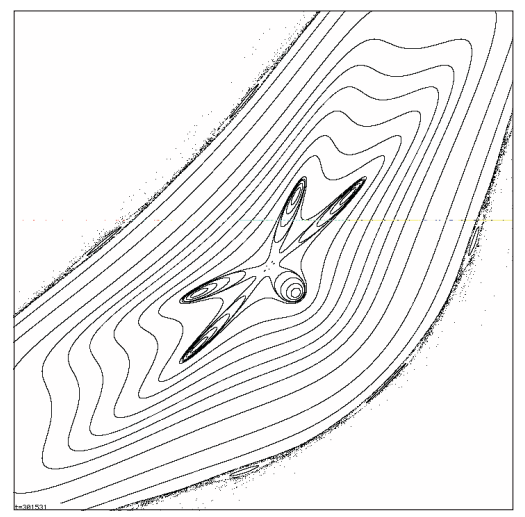

Figure 5: Phase space of $C_{-}$at the twistless bifurcation for $\omega=\frac{2}{5}$. Though the fixed point is unstable here, naive iteration is inconclusive: the central 5 points in the figure correspond to an orbit that was iterated $10^{7}$ times without substantially moving. Window bounds are $(0,-0.8) \times(0.8,0)$.

Since fixed points are also roots of $g_{2}$, we can can divide by the fixed point polynomial (24) to obtain

$$
p_{2}(x)=-4 \frac{g_{2}(x)}{g_{1}(x)}=p(x)^{2}+2 x p(x)+4 x^{2}-4 l-8,
$$

where we have used the explicit cubic form for $p$, and scaled by -4 for convenience. Since this polynomial has degree 6 , there at most 3 period 2 orbits. The discriminant of this polynomial gives the parameter values for which there are double roots, and corresponds to $t_{r}=2$ :

$$
\Delta_{2}(2)=\left(27 k^{2}-4(l+2)(l-4)^{2}\right)\left(27 k^{2}-4(l+4)^{3}\right)^{2} .
$$

The resulting curves are shown in Fig. 6. The curve of the first factor is the period doubling curve of the fixed point; it corresponds to a pitchfork bifurcation of the second iterate. The period two orbit is the child of a period doubling bifurcation, and therefore denoted by $c P D$. The supercritical case is denoted by $c P D^{p}$ and the subcritical case by $c P D_{b}$, see Fig. 6. The arrow indicates the direction in which the period two orbit is born. A similar notation is used for pitchfork bifurcations later on. The second factor is a saddle-node curve (because it appears squared in $\Delta_{2}$ ) and it is another Neils parabola with cusp at $(-4,0)$. The neighborhood of the cusp of this curve corresponds to a complex bifurcation. The Neils parabola is tangent to the $P D$ curve at $(l, k)=(-4 / 3, \pm 32 / 27 \sqrt{2})$, beyond which this curve actually marks a real $S N$ bifurcation of period two orbits. The tangency also has an important consequence for the interpretation of the $P D$ curve of the fixed point - not too surprisingly the tangency is the point where the fixed point does not have twist. The period doubling of the fixed point reverses its character at these points: for $l<-4 / 3$ it is subcritical, i.e. for increasing $l$ the unstable fixed point becomes stable and creates an unstable period 2 orbit. For $l>-4 / 3$ upon leaving the oval the standard, supercritical $P D$ occurs: the fixed point becomes unstable and creates a stable period 2 orbit. An example of this behavior is shown in Fig. 7 


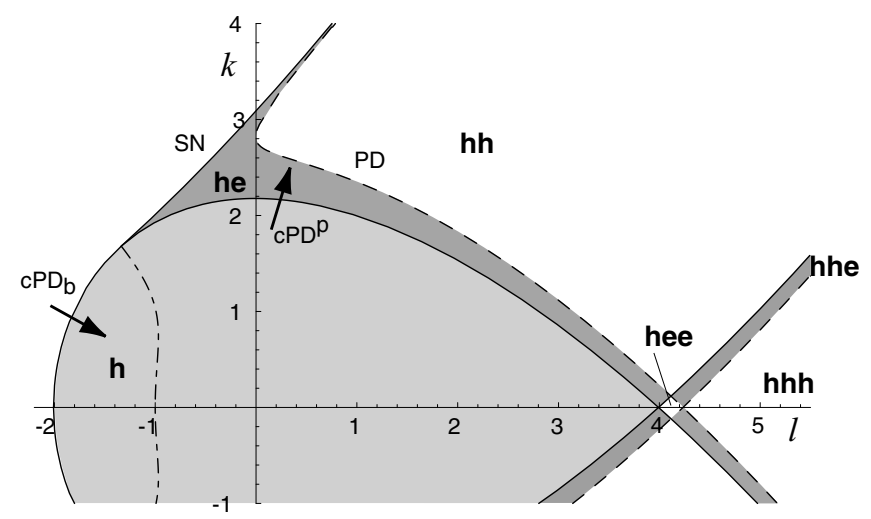

Figure 6: Bifurcation diagram for the period two orbits of $C_{-}$. The two solid curves correspond to $t_{r}=2$ for the second iterate. One is the saddle node $S N$ curve, $\Delta_{2}(2)=0$, and the other is the period doubling child $c P D$ curve, $\Delta_{1}(-2)=0$. The dashed curve is the $P D$ curve, $\Delta_{2}(-2)=0$, and the long-short dashed curve is the twistless curve of the fixed point.

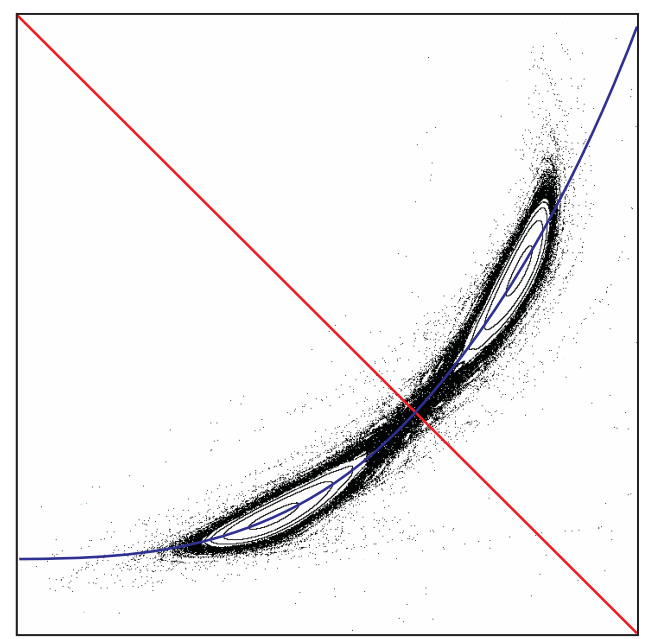

Figure 7: Phase space of $C_{-}$for $(l, k)=(-0.05,2.28)$ just above the supercritical $P D$ curve. The curves $\operatorname{Fix}(R)$ and $\operatorname{Fix}(R h)$ are also shown. Window bounds are $(0,-1.3) \times(1.3,0)$. 
The linear stability of the period two points can be calculated from the general formula (17). For a period two orbit we have $x_{1}=p\left(x_{0}\right) / 2$, where $x_{0}$ is given by a root of $p_{2}$. Since every period two orbit is symmetric, the complete orbit is $\left\{\left(x_{0},-p\left(x_{0}\right) / 2\right),\left(p\left(x_{0}\right) / 2,-x_{0}\right)\right\}$. Using this the trace of the orbit can be written as

$$
t_{r 2}=g_{2}^{\prime}\left(x_{0}\right)+2,
$$

similar to $(25)$ for the fixed point. Eliminating $x_{0}$ between this equation and $p_{2}\left(x_{0}\right)=0$ gives the resultant polynomial

$$
\begin{gathered}
\Delta_{2}\left(t_{r}\right)=729 k^{4}-27 k^{2}\left(408+186 l+24 l^{2}+8 l^{3}-12 t_{r}+3 l t_{r}\right) \\
+\left(34+24 l+4 l^{2}-t_{r}\right)\left(-34+2 l^{2}+t_{r}\right)^{2} .
\end{gathered}
$$

Because of the relation between $t_{r 2}$ and $g_{2}^{\prime}$ this reduces to the discriminant of $p_{2}$ when $t_{r}=2$. As usual, the curve $\Delta_{2}(-2)=0$ corresponds to period doubling bifurcation of the orbit; this curve is shown in Fig. 6. Numerically this appears to be a supercritical bifurcation, creating a stable period 4 orbit, but we have not analytically verified this.

As already described for arbitrary generalized Hénon maps the equations for symmetric orbits always reduce to a single polynomial. Each symmetric period 3 orbit has one point on $\operatorname{Fix}(R)$ and one point on $\operatorname{Fix}(R h)$, so we can look for points on $\operatorname{Fix}(R)$, where the equations reduce to

$$
g_{3 s}=p(p(x)-x)-2 x .
$$

This equation is of course divisible by the fixed point equation, so that we define

$$
p_{3 s}=-\frac{g_{3 s}(x)}{g_{1}(x)}=p(x)^{2}-x p(x)+x^{2}-l-1,
$$

where the second equality is only valid for the cubic map. This is a polynomial of degree 6 and each root $x_{0}$ corresponds to a distinct periodic orbit

$$
\left\{\left(x_{0},-x_{0}\right),\left(-x_{0}+p\left(x_{0}\right),-x_{0}\right),\left(x_{0}, x_{0}-p\left(x_{0}\right)\right)\right\} .
$$

In this way we find 6 symmetric period 3 orbits.

The $S N$ bifurcation curves of these orbits are obtained from the discriminant, $\Delta_{3 s}(2)$, of $p_{3 s}$. This expression (too large to be displayed here) gives four curves. Three are $S N$ curves shown in Fig. 8. The fourth corresponds to the tripling bifurcation $\Delta_{1}(-1)=0$ in which an unstable period three orbits collides with the fixed point. This accounts for the creation of six symmetric period three orbits.

Since there are $\left(3^{3}-3\right) / 3=8$ period 3 orbits there must be two nonsymmetric orbits; these are mapped into each other by $R$. To obtain these, we return to the general equations for a period three orbit, (16), which for $b=1$ become

$$
x_{0}+p\left(x_{0}\right)=x_{1}+p\left(x_{1}\right), \quad x_{1}+x_{0}=p\left(p\left(x_{1}\right)-x_{0}\right) .
$$

Using the resultant, $x_{0}$ can be eliminated from these equations and we obtain one polynomial in $x_{1}$. This polynomial factors, and apart from the known solutions, the fixed points and symmetric period three orbits, the remaining factor is surprisingly simple:

$$
p_{3 a}=p(x)+x .
$$




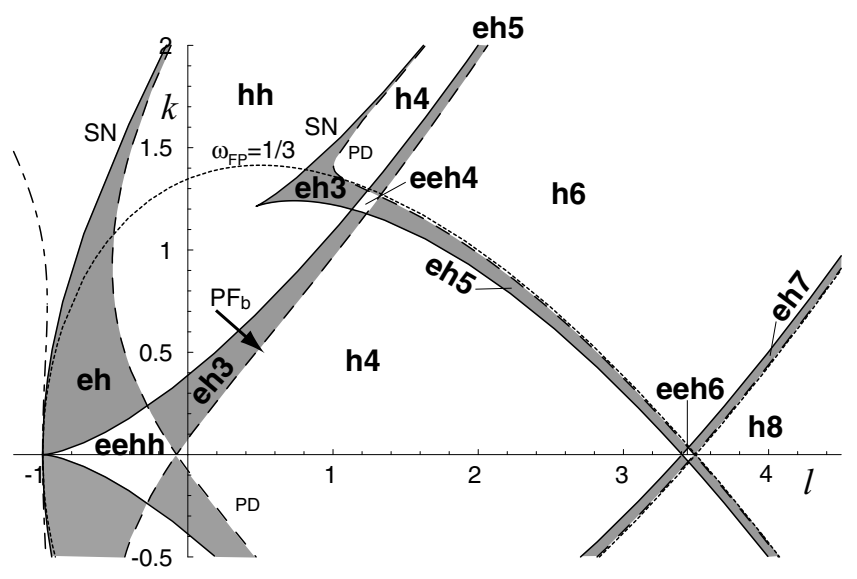

Figure 8: Bifurcation diagram for the period 3 orbits of $C_{-}$. Same dashing as in Fig. 1 now for the trace of $C_{-}^{3}$. The dotted curve is the tripling curve of a fixed point.

Denote the roots of this equation by $x_{0}, x_{1}$, and $x_{2}$. Then there are two orbits formed from these points. One is $\left\{\left(x_{0},-x_{2}\right),\left(x_{1},-x_{0}\right),\left(x_{2},-x_{1}\right)\right\}$ and the other one is obtained upon reflection by $R$. This accounts for the two asymmetric period 3 orbits, and in this way the union of the orbits is automatically invariant under $R$. The discriminant of $p_{3 a}$ is

$$
\Delta_{3 a}(2)=-27 k^{4}+4(l+1)^{3},
$$

which is another Neils parabola, with cusp at $(-1,0)$, see Fig. 8.

The stability of period 3 orbits is given by the general relation (18). In both the symmetric and asymmetric cases, we can eliminate the phase space coordinates from the expression for $t_{r}$. For example, in the asymmetric case the three coordinates are just the roots of $p_{3 a}$, so that we know the symmetric functions of $x_{0}, x_{1}$, and $x_{2}$. Since $t_{r 3}$ is also a symmetric function, the coordinates can easily be eliminated, giving

$$
\Delta_{3 a}\left(t_{r}\right)=-27 k^{2}+4(l+1)^{3}+2-t_{r}=\Delta_{3 a}(2)+2-t_{r} .
$$

Since the asymmetric orbits only exist when $\Delta_{3 a}(2) \geq 0$, we have $t_{r}>2$ whenever the asymmetric orbits exist, so that the pair of period 3 orbits created in the symmetry breaking pitchfork bifurcation is always unstable. Hence the bifurcation is subcritical, and we denote it $P F_{b}$.

For the symmetric orbits the calculation of the stability is also possible. Using the points of the symmetric orbit (27) in the general formula (18) we obtain the relation

$$
t_{r 3}\left(x_{0}\right)=g_{3 s}^{\prime}\left(x_{0}\right) p_{3 a}^{\prime}\left(x_{0}\right)+2 .
$$

In this case the relation is not simply given by the derivative of $g_{3}$ because there are also asymmetric orbits, which enter the formula even though we are currently calculating the stability of the symmetric orbits. As before we can eliminate $x_{0}$ between this equation and $p_{3 s}$, which gives the curve $\Delta_{3 s}\left(t_{r}\right)=0$, a huge polynomial in $k, l$, and $t_{r}$. By the 


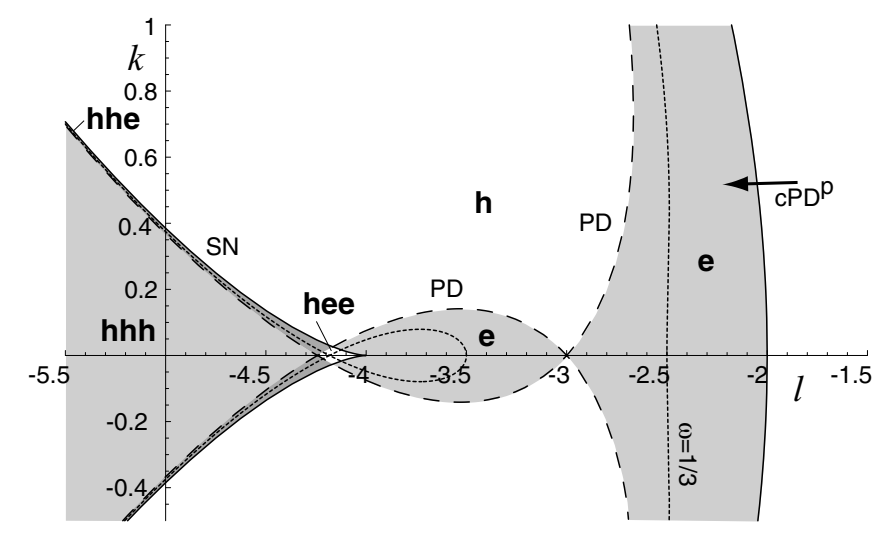

Figure 9: Bifurcation diagram for the period 2 orbits of $C_{+}$. The dashing is the same as in Fig. 1 applied to the trace of $C_{+}^{2}$.

above relation it reduces to the discriminant for $t_{r}=2$. The curve $\Delta_{3 s}(-2)=0$ on which the symmetric orbits have $t_{r}=-2$ has two factors one of which is given by

$$
\begin{aligned}
\Delta_{3 s}^{a}(-2)=729 k^{4}- & 27\left(8 l^{3}-12 l^{2}+45 l+67\right) k^{2}+ \\
& 4\left(2 l^{3}-3 l^{2}-9 l-17\right)^{2},
\end{aligned}
$$

while $\Delta_{3 s}^{b}(-2)$ is quartic in $k^{2}$ and too large to display. The curves $\Delta_{3 s}^{a}(-2)=0$ are asymptotic to the two cusped curves from $\Delta_{3 s}(2)$, see Fig. 8, the others are asymptotic to $\Delta_{3 a}(2)=0$ and the smooth curve from $\Delta_{3 s}=0$.

The bifurcation diagrams for these low period orbits have many features in common. In particular each $P D$ curve is asymptotic either to a $S N$ or a $P F$ curve as $(l, k)$ tend to infinity, so that even though there are stable orbits for arbitrarily large parameters, the domain of stability becomes extremely small. This is explained by the fact that the polynomials $\Delta\left(t_{r}\right)$ do not depend on $t_{r}$ in the leading order terms, so that asymptotically the difference between the curves $\Delta(2)=0$ and $\Delta(-2)=0$ vanishes.

\subsection{Periodic orbits for $C_{+}$}

The bifurcation curves for $C_{+}$can be obtained from those of $C_{-}$by the replacement $k \rightarrow i k$, because under the transformation

$$
x \rightarrow i x, \quad y \rightarrow i y, \quad k \rightarrow i k
$$

the mappings $C_{+}$and $C_{-}$are transformed into each other. We show the resulting bifurcation curves in Fig. 9 and Fig. 10. For the period 3 orbits of $C_{+}$there is one additional curve corresponding to $\Delta_{3 a}(-2)=0$. The main difference from $C_{-}$is that the bifurcation at $\Delta_{3 a}(-2)=0$ is now of type $P F^{p}$, so that the newly created orbits are stable until they period double when $\Delta_{3 a}(-2)=0$, see Fig. 10 .

There is one new codimension two bifurcation of periodic orbits of $C_{+}$. Consider first the period 2 orbit, Fig. 9. The boundaries of the domains in which there is a single stable period two orbit touch at the point $(l, k)=(-3,0)$, which is the intersection of two period doubling curves. At this point two stable, period four orbits are simultaneously created, 


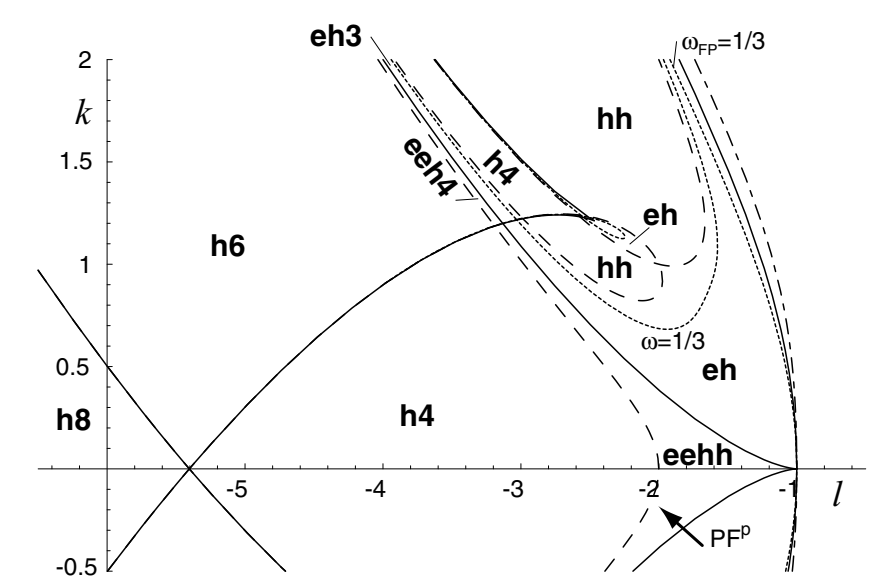

Figure 10: Bifurcation diagram for the period 3 orbits of $C_{+}$. Dashing as in Fig. 1 but for $C_{+}^{3}$. Here the shading is omitted because of the extremely small regions between $P D$ and $S N$ curves. The dotted lines (tripling) do not constitute boundaries of regions with the same number of elliptic/hyperbolic periodic orbits, all others do.

and the period two orbit is unstable; we denote this bifurcation by $P D^{p p}$. An apparently similar crossing of two $P D$ curves also appears in the same figure for $l=-4.25$ and also in Fig. 6 at $l=3 \sqrt{2} \approx 4.24$. In these cases, however, the two period doublings occur for different orbits, so that from the point of view of bifurcation theory these crossings are incidental. Another easy way to recognize this bifurcation is to note that the number of elliptic orbits in the four regions touching an incidental crossing has the pattern 1,0,1,2 and not, as for $P D^{p p}$, the pattern 1,0,1,0.

A different codimension two $P D$ bifurcation occurs for period 3, where the two period doubling curves intersect at $(l, k)=(-2,1)$. In this case there is also a pitchfork bifurcation of period 6 orbits at this point, which gives rise to a pair of asymmetric period 6 orbits in some sectors of the bifurcation diagram. We show the phase portraits near the codimension two point in Fig. 12. Here we show only one point on the period 3 orbit. The period doubling bifurcations change from supercritical to subcritical as we pass through the codimension two point, so we can denote this bifurcation by $P D_{b}^{p}$. This bifurcation does not occur for period 3 orbits of $C_{-}$; the crossings of the period doubling curves in Fig. 8 are incidental.

Another way to distinguish between the two types of crossings of $\Delta(-2)=0$ curves is to consider nearby curves of elliptic orbits $\Delta(-2+\epsilon)=0$. If those also intersect it is an incidental intersection, if they don't then it is a codimension 2 bifurcation. To illustrate this, the tripling curves of the periodic orbits are also shown in Fig. 9 and Fig. 10. Compare these to Fig. 2 where the tripling curves do intersect close to the point where the doubling curves intersect at $(4,0)$.

\subsection{AIL}

The cubic map is in anti-integrable form (19) if we choose $U^{\prime}(x)=k m^{3 / 2}+\operatorname{lm} x \pm x^{3}$. The potential $U$ has three critical points when $\Delta=4|l|^{3}-27 k^{2}>0$, and $\operatorname{sgn}(l)=\mp 1$. So for a nontrivial anti-integrable limit in the $+x^{3}$ case we want to approach $\infty$ to the left of the 


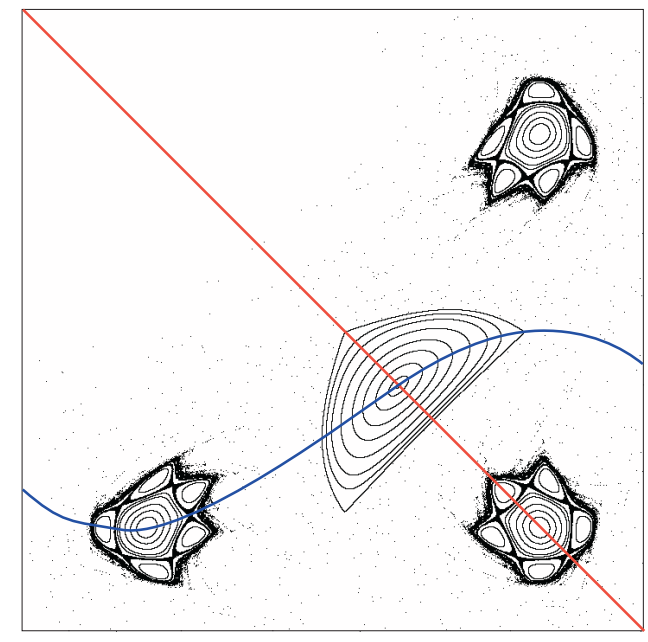

Figure 11: Phase space of the map $C_{+}$at $(l, k)=(-1.4,0.7)$ after the tripling bifurcation of the elliptic fixed point. Window bounds are $(-1,-1) \times(1,1)$.

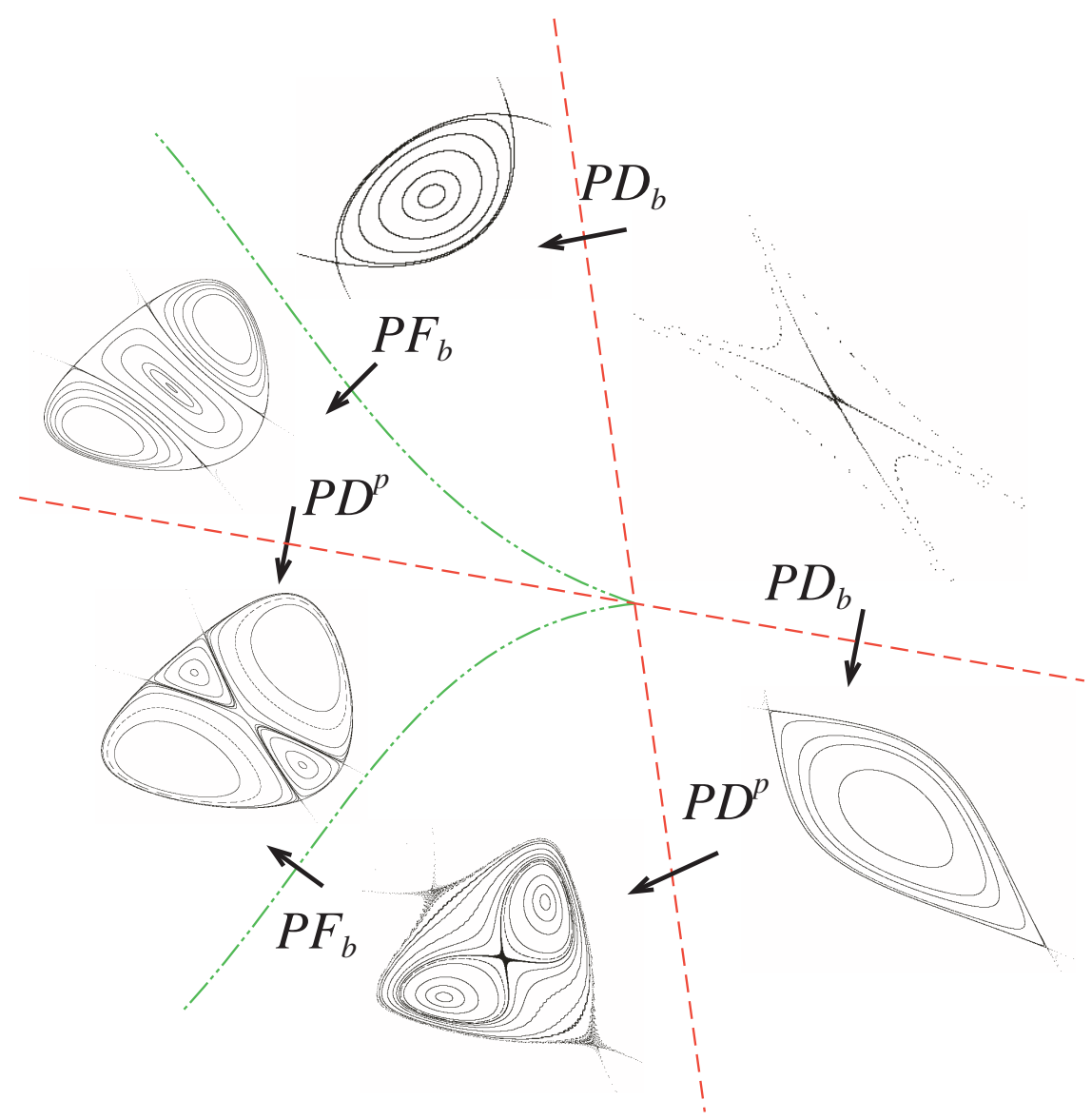

Figure 12: Phase portraits near the codimension two point $(-2,1)$ for $C_{+}$. The bifurcation corresponds to the crossing of two period doubling curves for the period three orbit. The phase portraits show one of the points on this orbit, and nearby period six orbits created either in period doubling or pitchfork bifurcations. 


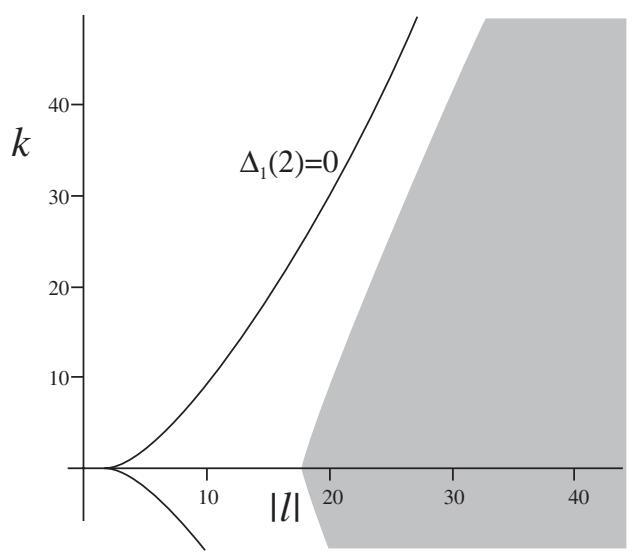

Figure 13: Parameter region for which there is a hyperbolic three symbol horseshoe for the areapreserving cubic maps $C_{ \pm}$. The horseshoe exists in the shaded region, when we take $\operatorname{sgn}(l)=\mp 1$.

Neils parabola in the bifurcation diagram Fig. 1 (which asymptotically approaches the discriminant $\Delta$ ); while for $-x^{3}$, we want to approach $\infty$ to the right of the Neils parabola in Fig. 2.

The bound (22) can be made more explicit for this case

Theorem 9. Let $L=|l| /(1+|b|)$, where $\operatorname{sgn}(l)=\mp 1$. Suppose that $L>(6+2 \sqrt{2})$ and

$$
|k|<\left(\frac{|l|}{2}\right)^{3 / 2} \frac{(L-2)\left(L^{2}-12 L+28\right)}{(L-4)^{3}},
$$

then the dynamics of the bounded orbits of the cubic map $C_{ \pm}$is conjugate to a full shift on three symbols.

Proof. To obtain this bound from (22), we choose $\alpha=|m l| / 2$, The three roots of the cubic $U^{\prime}$ can be written $z_{j}= \pm 2 \operatorname{sgn}(k) \sqrt{\frac{|l m|}{3}} \cos (\phi-2 j \pi / 3)$, where $\phi=\pi-\frac{1}{3} \arctan \left(\sqrt{\frac{\Delta}{27 k^{2}}}\right)$. Thus the largest root is $Z=\left|z_{0}\right|$. Some elementary manipulations of (21) and (22) give the result

$$
L>2\left(1+\frac{|\cos \phi|}{\cos (\phi-2 \pi / 3)-\sqrt{\frac{3}{8}}}\right)
$$

Solving this for $k$ and keeping track of the absolute values yields (28).

This bound is shown in Fig. 13 for the area-preserving case. When $k=0,(28)$ gives $|l|>2(1+|b|)(3+\sqrt{2}) \approx 8.83(1+|b|)$. This bound is not especially strict; for example, in Fig. 14 we show the creation of the horseshoe for $C_{+}$, near $(-6.6,1)$. When $l=-5$, the horseshoe is incomplete, as shown by the iteration of the square in the figure.

\section{Conclusions}

The study of generalized Hénon maps is of interest because any nontrivial polynomial diffeomorphism of $\mathbb{R}^{2}$ with constant Jacobian is equivalent to a composition of generalized 

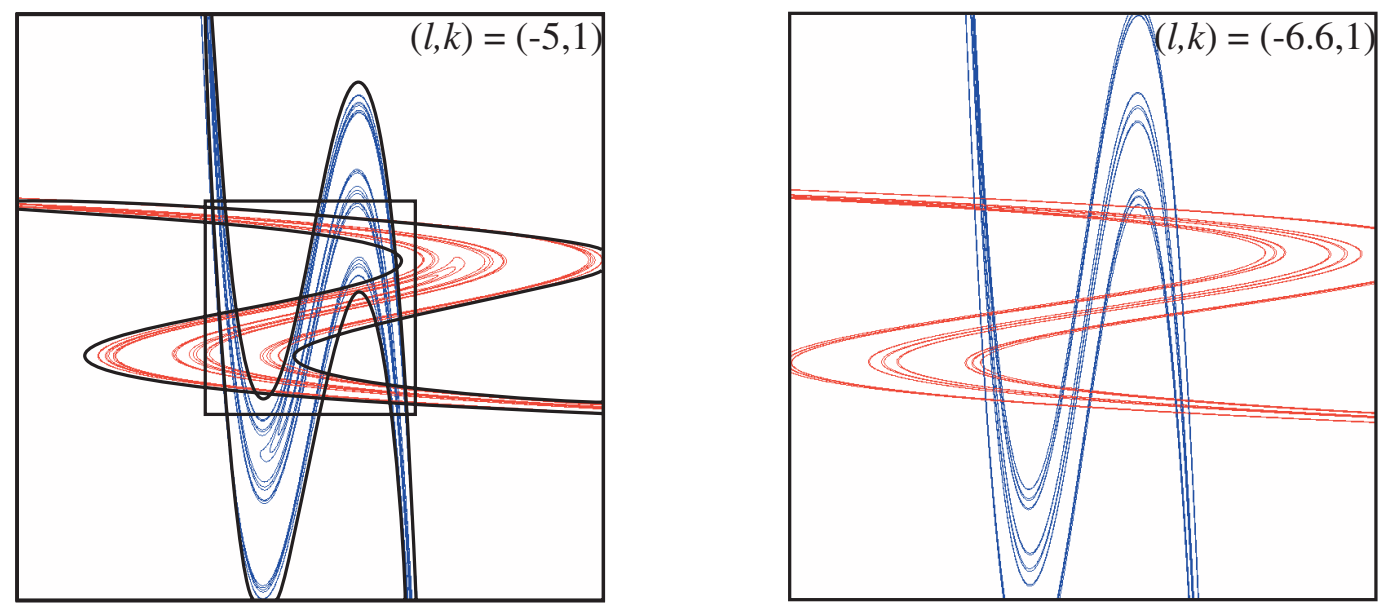

Figure 14: Creation of the horseshoe for $C_{+}$. Each panel shows the stable and unstable manifolds of one of the saddle fixed points. The left panel also shows the image and preimage of the square with corners $(-2.86,-2.86) \times(2.86,2.86)$.

Hénon maps. Note that a complex analytic polynomial map must have constant Jacobian in order to be a diffeomorphism, since any nonconstant polynomial has roots. However, the restriction to constant Jacobian is necessary for the real case.

The simplest case (length 2) is a single Hénon map, $h$. For the area-preserving case, $h$ has the special property of being reversible. When the critical points of the polynomial in $h$ are nondegenerate, $h$ has an anti-integrable limit, and a corresponding domain in parameter space for which there is a hyperbolic horseshoe. By contrast, when $h$ has an elliptic fixed point, we used the Birhkoff normal form to study its stability.

By studying the bifurcations of orbits of the cubic Hénon maps $C_{ \pm}$, we have classified bifurcations for all cubic area-preserving maps. These are reversible and have an additional symmetry on a codimension one line in parameter space. Therefore this study also shows how the bifurcations of a system with two reversors unfolds into the more generic case of only one reversor.

We have found a number of codimension 2 bifurcations in the cubic family. The twistless bifurcations are predicted by the general theory in [15]. The consequence of such bifurcations is the nonmonotonicity of the rotation number as a function of distance from the periodic orbit. This typically results in reconnection bifurcations and meandering invariant curves. The codimension 2 period doubling bifurcations, corresponding to the crossing of two period doubling curves organize other bifurcations such as the pitchfork bifurcations shown in Fig. 12. It would be interesting to study the generic unfolding of this situation.

The only drawback from the general point of view is the presence of the reversor. As we already argued it would be necessary to study maps of degree 4 and length 4 to have the generic case. It will be interesting to study the bifurcations in this three parameter family of generic area preserving maps. 


\section{Acknowledgments}

One of us (HRD) would like to thank A.P. Veselov for useful discussions.

\section{References}

[1] M. Hénon. A two-dimensional mapping with a strange attractor. Comm. Math. Phys, 50(1):69-77, 1976.

[2] Etienne Forest. Beam Dynamics : A New Attitude and Framework (The Physics and Technology of Particle and Photon Beams). Harwood Academic, Amsterdam, 1998.

[3] Alex J. Dragt and Dan T. Abell. Symplectic maps and computation of orbits in particle accelerators. In Integration algorithms and classical mechanics (Toronto, ON, 1993), pages 59-85. Amer. Math. Soc., Providence, RI, 1996.

[4] G. Meisters. Annotated bibliography on polynomial maps. Technical report, Univ. of Nebraska, June 1996. http://www.math.unl.edu/ gmeister/.

[5] H.W.E. Jung. Uber ganze birationale Transformationen der Ebene. J. Reine Angew. Math., 184:161-174, 1942.

[6] S. Friedland and J. Milnor. Dynamical properties of plane polynomial automorphisms. Ergod. Th. E Dyn. Systems, 9:67-99, 1989.

[7] H. Bass, E.H. Connell, and D. Wright. The Jacobian conjecture: Reduction of degree and formal expansion of inverse. Bull. Amer. Math. Soc., 7:287-330, 1982.

[8] H.P. Hudson. Cremona Transformations in Plane and Space. Cambridge University Press, London, 1927.

[9] S. Wang. A Jacobian criterion for separability. J. Algebra, 65:453-494, 1980.

[10] A. van den Essen. Seven lectures on polynomial automorphisms. In A. van den Essen, editor, Automorphisms of Affine Spaces, pages 3-40, Dordrecht, 1995. Kluwer Academic.

[11] W. Rudin. Injective polynomial maps are automorphisms. Amer. Math. Monthly, 106(6):540-543, 1995.

[12] J.H. McKay and S.S-S Wang. An elementary proof of the automorphism theorem for the polynomial ring in two variables. J. Pure Appl. Algebra, 52:91-102, 1988.

[13] W. Engel. Ganze Cremona-Transformationen von Prinzahlgrad in der Ebene. Math. Ann., 136:319-325, 1958.

[14] J.S.W.Lamb, editor. Time-Reversal Symmetry in Dynamical Systems, volume 112 of Physica D, Amsterdam, 1998. Elsevier. 
[15] H.R. Dullin, J.D. Meiss, and D. Sterling. Generic twistless bifurcations. Nonlinearity, 1999.

[16] C.L. Siegel and J.K. Moser. Lectures on Celestial Mechanics. Classics in Mathematics. Springer-Verlag, New York, 1971.

[17] V.I. Arnold, editor. Dynamical Systems III, volume 3 of Encyclopaedia of Mathematical Sciences. Springer Verlag, New York, 1988.

[18] J.D. Meiss. Symplectic maps, variational principles, and transport. Reviews of Modern Physics, 64(3):795-848, 1992.

[19] S. J. Aubry. Anti-integrability in dynamical and variational problems. Physica D, 86:284-296, 1995.

[20] R.S. MacKay and J.D. Meiss. Cantori for symplectic maps near the anti-integrable limit. Nonlinearity, 5:149-160, 1992.

[21] D. Sterling and J.D. Meiss. Computing periodic orbits using the anti-integrable limit. Physics Letters A, 241:46-52, 1998.

[22] M. Barge and J Franks. Recurrent sets for planar homeomorphisms. In From Topology to Computation: Proceedings of the Smalefest, pages 186-195, New York, 1993. Springer.

[23] R.S. MacKay. Equivariant universality classes. Phys. Lett. A, 106:99-100, 1984.

[24] H.E. Cabral and K.R. Meyer. Stability of equilibria and fixed points of conservative systems. Nonlinearity, 12:1351-1362, 1999. 